From: "Handbook of Liquid Crystals, Volume 2: Physical properties

and phase behavior of liquid crystals" (2nd edition),

edited by J.W. Goodby, P.J. Collings, T. Kato, C. Tschierske,

H.F. Gleeson and P. Raynes

(Wiley-VCH, Weinheim, Germany 2014).

[copyright Wiley: only for personal use]

2

\title{
Magnetic Properties of Liquid Crystals
}

David Dunmur, Malgosia Kaczmarek, and Tim Sluckin

2.1

Introduction

In 1888, Friedrich Reinitzer [1] first observed an anomalous turbid fluid phase in cholesteryl benzoate. This phase interposed itself between a crystal and a normal clear fluid phase. This fluid was later to be identified as the first liquid crystal. After some years of study, it became clear that the turbidity was not due to density inhomogeneity, as would normally be supposed in such a situation, but rather due to inhomogeneities in the principal optical axis in an optically anisotropic fluid. Thus a key problem in studying what were at that time often labeled as Lehmann's liquid crystals was to obtain a uniform optical alignment.

In 1911, Charles Mauguin [1], working with azoxyanisole and azoxyphenetole, found that magnetic fields aligned liquid crystals in situations when prepared boundaries alone were unable to do so. Mauguin's fields - up to about $0.75 \mathrm{~T}$ - even competed with boundary alignment in such a way that the principal optic axis could sometimes be tilted with respect to the cell. Further investigations by Ornstein and coworkers in the Netherlands in the 1920s showed that magnetic fields (in modern language) reduced the orientational correlation lengths, although these results were originally interpreted in terms of an incorrect theory.

More extensive investigations by Frederiks in the Soviet Union in the 1920s $[1,2]$, working with magnetic fields up to $2.5 \mathrm{~T}$, showed that a given magnetic field was able to align a thick liquid crystalline cell, but failed to align a thinner cell. This is the familiar Frederiks effect. Zocher [1], following an elastic theory originally introduced by Oseen [1], introduced the term coupling the magnetic field to what later became known as the nematic director. By doing so, he was able to fit Frederiks's results to Oseen's theory, thereby establishing the elastic theory as the liquid crystal theory of choice. Nowadays, the aligning field is usually electric, and the field, rather than the cell thickness, is varied. The physics is the same, but the response to easily accessible electric fields is much greater than to magnetic fields. The question of increasing the magnetic sensitivity has become a subject of interest in recent years because of its device implications, and we return to this problem later in this chapter. 
In modern times, magnetic fields played a major role in both the de Gennes statistical mechanical theory [3] and the Leslie-Ericksen hydrodynamical theory [4]. de Gennes pointed out that the magnetic birefringence in an isotropic fluid (the Cotton-Mouton effect) would be much larger in mesogenic materials; the susceptibility would increase toward the onset of the nematic orientationally ordered phase, diverging at a temperature $T^{*}$ just below the nematic-isotropic temperature $T_{\mathrm{NI}}$. Leslie [5], in work separate from the hydrodynamic theory, considered the magnetic Frederiks threshold in a cell with a twisted geometry, thereby unwittingly and accidentally providing a theory for the nascent twisted nematic display device.

The macroscopic anisotropy in liquid crystal properties can be related to molecular properties through appropriate microscopic orientational order parameters. The relationship assumes that the macroscopic response of a liquid crystal is simply the sum of the individual molecular responses averaged over an orientational distribution function, that is, interactions between molecules are ignored, except to the extent that they determine the orientational order. For most physical properties such an approximation is very crude; however, magnetic properties are only very weakly influenced by intermolecular interactions, at least for nonferromagnetic materials, and so it can be assumed that the magnetic response of liquid crystals is simply the aggregated molecular response. The weak interaction between molecules and magnetic fields is evidenced by the magnetic permeability relative to that of free space for nonferromagnetic materials, which is close to unity. The magnetic response of materials depends on their electronic structure, and the susceptibility may be negative, characteristic of diamagnetic compounds, or positive, denoting a paramagnetic response.

Most liquid crystals are diamagnetic (zero net-spin) and their magnetic properties have been evaluated for a number of materials. The values of liquid crystal diamagnetic susceptibilities are low, but the diamagnetic response of both nematic and smectic liquid crystals to magnetic fields is a valuable experimental tool in evaluating many properties of liquid crystals. Given the variety of structures that can form liquid crystals, an important aspect of their magnetic properties is the influence of different terminal substituents and core structures on the magnitude of magnetic anisotropy. This aspect is dealt with in detail for diamagnetic liquid crystals in Chapter 7 by R. Stannarius included in this Handbook (Part II, Volume 3).

Paramagnetic liquid crystals can be formed by incorporating an electron-spinbearing metal atom, molecule, or ion into the mesogenic structure, and there are now a number of systems that form paramagnetic liquid crystals. For liquid crystal properties and applications, it is usually the anisotropy of the magnetic properties that is important, and the enhancement of this in liquid crystal-forming molecules (mesogens) has presented a number of challenges. Paramagnetic mesogens incorporating a variety of metal centers have been prepared and their magnetic properties examined. Of particular interest are mesogens containing lanthanide metals (rare earths) with $4 \mathrm{f}$ electrons, which can result in very high values of paramagnetic susceptibility anisotropy. 
Ferromagnetic liquid crystals form a class apart from diamagnetic and paramagnetic liquid crystals. In general, ferromagnetism is exhibited by materials in which a permanent magnetization results from coupling between electron spins on constituent atoms, ions, or molecules. Thus ferromagnetism is largely restricted to the solid state. True ferromagnetic liquid crystalline materials appear to be rare, although a ferromagnetic liquid crystal material has recently been claimed (see Section 2.8.2 [6]) by intercalating an iron compound into a discotic liquid crystal. An alternative route to liquid crystal ferromagnetism lies in the suggestion by Brochard and de Gennes [7] that, effectively, ferromagnetic liquid crystals could be prepared using colloidal suspensions of ferromagnetic materials in a liquid crystal host.

We note that the synthesis of ferromagnetic liquid crystals has long been a research goal, with both fundamental and technical motivations. The basic physics motivation lies in a folk belief that liquid disorder would counteract any ferromagnetism, and thus the detection of liquid crystalline ferromagnetism would show that this is incorrect. In fact, ferromagnetic liquid crystals would be expected to show a rich set of behaviors in which the magnetization and the nematic director would interact. The technical reason for seeking ferromagnetic liquid crystals is more prosaic; a linear rather than a quadratic interaction with a magnetic field might be expected dramatically to enhance the nematic susceptibility to low fields, thus making practical the idea of a magnetically based liquid crystal device.

In this chapter, we present the basic principles of magnetism as they apply to the magnetic properties of liquid crystals. The layout of this chapter is as follows. In Section 2.2, we outline key formulae related to magnetic anisotropy. In Section 2.3, we discuss types of magnetic polarization. In Section 2.4, we discuss magnetic field effects in liquid crystals. In Section 2.5, we treat the Frederiks effect; this was originally a magnetic effect, although in its present technological context, it is usually thought of as an electric field effect. In Section 2.6 and Section 2.7, we discuss diamagnetic and paramagnetic liquid crystals. Finally, in Section 2.8, we examine recent work on ferromagnetism in liquid crystals, paying particular attention to ferronematic materials, which are colloidal suspensions of ferromagnetic particles in liquid crystal matrix. An important discussion about magnetic units is included in the Appendix. Some other results of interest, in a magnetic context, are presented in more detail in other chapters.

\section{2}

\section{Magnetic Anisotropy}

Similar to other tensor properties of liquid crystals, magnetic susceptibility is anisotropic, and so magnetic fields can be used to control the alignment of liquid crystal samples. This is perhaps the single most useful application of the magnetic properties of liquid crystals, and the combination of magnetic field alignment with some other measurement forms the basis of many experimental investigations. 
Macroscopically, the magnetic susceptibility relates the induced magnetization $M$ to the strength of the magnetic field, but assuming that local field effects are ignored, the susceptibility is usually defined in terms of magnetic induction $B$. The magnetization is given by

$$
M_{\alpha}=\mu_{0}^{-1} \chi_{\alpha \beta}^{\mathrm{mag}} B_{\beta}
$$

where $\mu_{0}$ is the permeability of free space. The magnetic contribution to the free energy density becomes

$$
\begin{aligned}
g_{\text {mag }} & =-\int B_{\alpha} \mathrm{d} M_{\alpha}=-\mu_{0}^{-1} \int B_{\alpha} \chi_{\alpha \beta} \mathrm{d} B_{\beta} \\
& =g_{0}-\frac{1}{2} \mu_{0}^{-1} \chi_{\alpha \beta} B_{\alpha} B_{\beta}
\end{aligned}
$$

$\chi_{\alpha \beta}$ is a volume susceptibility, but a molar susceptibility $\chi_{\alpha \beta}^{\text {mol }}$ may be defined as

$$
\chi_{\alpha \beta}^{\mathrm{mol}}=\chi_{\alpha \beta} V^{\mathrm{mol}}
$$

where $V^{\text {mol }}$ is the molar volume. The susceptibility has the symmetry of the material, so expressing this in terms of the principal axes of $\chi$ gives for the free energy density

$$
\begin{aligned}
g_{\text {mag }} & =g_{0}-\frac{1}{2} \mu_{0}^{-1}\left(\chi_{\|} B_{\|}^{2}+\chi_{\perp^{\prime}} B_{\perp^{\prime}}^{2}+\chi_{\perp} B_{\perp}^{2}\right) \\
& =g_{0}-\frac{1}{2} \mu_{0}^{-1} B^{2}\left(\chi_{\|} \cos ^{2} \theta+\chi_{\perp^{\prime}} \sin ^{2} \theta \sin ^{2} \phi+\chi_{\perp} \sin ^{2} \theta \cos ^{2} \phi\right)
\end{aligned}
$$

where $\chi_{\|}, \chi_{\perp}$ and $\chi_{\perp}$ are the principal components of the susceptibility tensor, and $\theta$ and $\phi$ are angles defining the orientation of $\mathbf{B}$ with respect to the principal axes of the susceptibility. For a uniaxial material $\chi_{\perp^{\prime}}=\chi_{\perp}$ and

$$
\begin{aligned}
g_{\text {mag }} & =g_{0}-\frac{1}{2} \mu_{0}^{-1} B^{2}\left(\chi_{\perp}+\Delta \chi \cos ^{2} \theta\right) \\
& =g_{0}-\frac{1}{2} \mu_{0}^{-1} B^{2} \chi_{\perp}-\frac{1}{2} \mu_{0}^{-1} \Delta \chi(\mathbf{B} \cdot \mathbf{n})^{2}
\end{aligned}
$$

where $\Delta \chi=\chi_{\|}-\chi_{\perp}$, and $\mathbf{n}$ is the director, which also defines the principal axis of the susceptibility for uniaxial materials. From Equation 2.5, it is clear that the sign of the anisotropy $\Delta \chi$ will determine the orientation of the director with respect to a magnetic field. In order to minimize the free energy, the director will align parallel to the magnetic field for a material having $\Delta \chi$ positive, while for $\Delta \chi$ negative the director will be perpendicular to $\mathbf{B}$; both situations are realized in practice.

Any anisotropic property can be used to define a macroscopic order parameter, and because it is largely unaffected by molecular interactions, the magnetic susceptibility is a particularly useful measure. The value of $\Delta \chi_{\max }$ corresponding to perfect alignment can, in principle, be obtained from measurements on single crystals or from molecular susceptibilities. The molecular susceptibilities $\kappa_{x x}, \kappa_{y y}$, $\kappa_{z z}$ are defined for the principal axes of the molecular susceptibility, which may not coincide with the axes that define the local orientational order. Measurements 
of the magnetic susceptibility can provide a useful route to the order parameters of liquid crystals [8,9], but require a knowledge of the molecular susceptibilities. These are not usually available for mesogens, but they can be obtained from singlecrystal measurements, provided full details are available for the crystal structure; the method for deriving molecular susceptibilities from crystal susceptibilities is explained in detail in [10].

\section{3}

\section{Types of Magnetic Polarization}

\subsection{1}

\section{Diamagnetism}

A diamagnetic response is the induction of a magnetic moment in opposition to an applied magnetic field, which thereby raises the free energy. Thus a diamagnetic material will be expelled from a magnetic field or will adjust itself to minimize the diamagnetic interaction. Most liquid crystals are diamagnetic and this diamagnetism originates from the dispersed electron distribution associated with the molecular electronic structure. The diamagnetic susceptibility is a second-rank tensor, and its principal components can be expressed as

$$
\begin{aligned}
\kappa_{l l}^{\mathrm{dia}} & =-\frac{e^{2} \mu_{0}}{4 m_{\mathrm{e}}}\left\langle m^{2}+n^{2}\right\rangle \\
\kappa_{m m}^{\mathrm{dia}} & =-\frac{e^{2} \mu_{0}}{4 m_{\mathrm{e}}}\left\langle l^{2}+n^{2}\right\rangle \\
\kappa_{n n}^{\mathrm{dia}} & =-\frac{e^{2} \mu_{0}}{4 m_{\mathrm{e}}}\left\langle m^{2}+l^{2}\right\rangle
\end{aligned}
$$

$e$ is the electronic charge, $m_{\mathrm{e}}$ is the mass of an electron, $l, m, n$ are the molecular axes, and the quantities such as $\left\langle m^{2}+n^{2}\right\rangle$ are averages over the electron distribution for a plane perpendicular to the component axis ( $l$ in this case; see Figure 2.1 ).

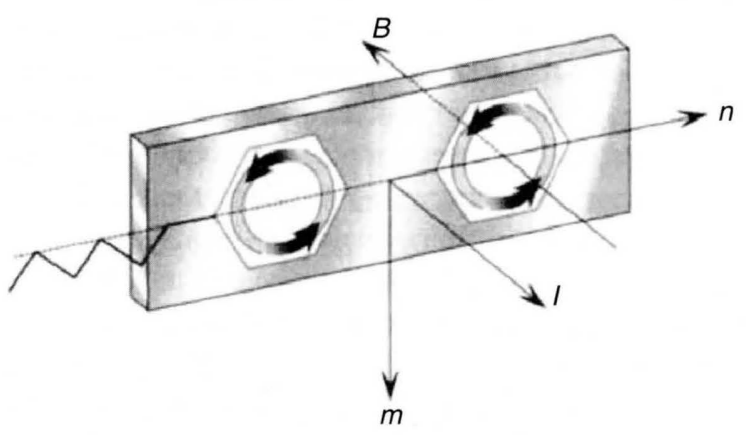

Figure 2.1 Diagram of molecular axes with representation of perpendicular plane. 
The induced diamagnetic moment depends on the extent of the electron distribution in a plane perpendicular to an applied magnetic field. In a molecule, delocalized charge makes a major contribution to $\kappa^{\text {dia }}$, and in particular, the ring currents associated with aromatic units give a large negative component of diamagnetic susceptibility for directions perpendicular to the plane of the aromatic unit. It is for this reason that the diamagnetic anisotropy of most calamitic mesogens is positive as both components are negative, but $\left|\chi_{\perp}\right|>\left|\chi_{\|}\right|$.

\subsection{2}

\section{Paramagnetism}

Molecular paramagnetism is mostly connected with unpaired electron spins, which have associated magnetic moments. For paramagnetic mesogens, the electron spin is introduced by metal centers, and one of the motivations for research into metal-containing mesogens is the desire to prepare paramagnetic liquid crystals. Orientation by an external magnetic field of the magnetic moment derived from an electron spin will induce a magnetization along the field direction and so provides a positive contribution to the magnetic susceptibility

$$
\bar{\kappa}^{\text {para }}=\frac{\mu_{0} \mathrm{~g}_{\mathrm{e}}^{2} \mu_{\mathrm{B}} s(s+1)}{3 k_{\mathrm{B}} T}
$$

where $g_{\mathrm{e}}$ is the electronic $g$-value, $s$ is the total electron spin quantum number, and $\mu_{\mathrm{B}}$ is the Bohr magneton. $\bar{\kappa}^{\text {para }}$ is the isotropic molecular paramagnetic susceptibility, and the coefficient of $1 / 3$ arises from the isotropic average of $\left\langle\cos ^{2} \theta\right\rangle$ over the spin orientations in a magnetic field. The temperature dependence of $\bar{\kappa}^{\text {para }}$ follows a simple Curie law, and as written, there is no anisotropy in $\bar{\kappa}^{\text {para }}$, as in most molecules, the unpaired electron spin is decoupled from the molecular structure and will align with an external magnetic field independently of the orientation of the molecule.

In addition to the magnetic moment from electron spin, there is another source of magnetization from the magnet moment associated with the orbital angular momentum of electrons in atoms and molecules. Interaction between the spin angular momentum and the orbital angular momentum, known as spin-orbit coupling, causes the effective magnetic moment to be coupled to the molecular frame, and hence leads to magnetic anisotropy for liquid crystals. Mostly, this is a rather small effect, but for molecules, or, in our case, mesogens, containing metal atoms with $4 \mathrm{f}$ electrons (lanthanides or rare earths), spin-orbit coupling can be very strong. There are two important physical consequences of strong spin-orbit coupling in lanthanides: one is enhanced photoluminescence from molecules containing lanthanides and the other is a strong magnetic anisotropy for certain of the lanthanide-containing molecules.

Anisotropy in $\bar{\kappa}^{\text {para }}$ can be introduced if the g-value becomes anisotropic. The g-value is a tensor quantity that describes the modification to the magnetic field experienced by the electron spin arising from the electron distribution in the 
molecule. It is analogous to the nuclear shielding in nuclear magnetic resonance and it contributes to the magnetic internal energy as

$$
u_{\text {mag }}=-\mathrm{g}_{\alpha \beta}^{\mathrm{e}} \gamma_{\mathrm{e}} s_{\alpha} B_{\beta}
$$

where $\gamma_{\mathrm{e}}$ is the electronic gyromagnetic ratio and $s_{\alpha}$ is the vector component of the electronic spin.

For nonlanthanide paramagnetic liquid crystals, which contain metals such as $\mathrm{Cu}(\mathrm{II}), \mathrm{V}(\mathrm{IV}) \mathrm{O}$, and $\mathrm{Fe}(\mathrm{III})$, as well as simple ions and radicals, the spin-orbitinduced anisotropy is quite small, and the principal components of the g-tensor usually differ by only small amounts from the free-electron value of $g=2.0023$. For such systems, the principal components of the paramagnetic susceptibility can be written as

$$
\kappa_{i i}^{\text {para }}=\frac{\mu_{0}\left(g_{i i}^{\mathrm{e}}\right)^{2} \mu_{\mathrm{B}}^{2} s(s+1)}{3 k_{\mathrm{B}} T}
$$

For a uniaxial liquid crystal, and neglecting any biaxial local order, the principal components of the molar paramagnetic susceptibility become

$$
\begin{aligned}
& \chi_{\|}^{\text {para }}=\bar{\chi}+\frac{2 N \mu_{0} \mu_{\mathrm{B}}^{2} s(s+1) S}{3 k_{\mathrm{B}} T}\left[\left(\mathrm{~g}_{n n}^{\mathrm{e}}\right)^{2}-\frac{1}{2}\left\{\left(\mathrm{~g}_{l l}^{\mathrm{e}}\right)^{2}+\left(\mathrm{g}_{m m}^{\mathrm{e}}\right)^{2}\right\}\right] \\
& \chi_{\perp}^{\text {para }}=\bar{\chi}-\frac{N \mu_{0} \mu_{\mathrm{B}}^{2} s(s+1) S}{3 k_{\mathrm{B}} T}\left[\left(\mathrm{~g}_{n n}^{\mathrm{e}}\right)^{2}-\frac{1}{2}\left\{\left(\mathrm{~g}_{l l}^{\mathrm{e}}\right)^{2}+\left(\mathrm{g}_{m m}^{\mathrm{e}}\right)^{2}\right\}\right]
\end{aligned}
$$

where $N$ is Avogadro's number. For a uniaxial $\mathbf{g}_{\mathrm{e}}$ tensor, the paramagnetic susceptibility anisotropy and mean susceptibility can be written as

$$
\begin{aligned}
& \Delta \chi^{(\mathrm{a}) \text { para }}=\frac{N \mu_{0} \mu_{\mathrm{B}}^{2} s(s+1) S}{k_{\mathrm{B}} T}\left[\left(g_{n n}^{\mathrm{e}}\right)^{2}-\left(g_{l l}^{\mathrm{e}}\right)^{2}\right] \\
& \bar{\chi}^{\text {para }}=\frac{N \mu_{0} \mu_{\mathrm{B}}^{2} s(s+1)}{9 k_{\mathrm{B}} T}\left[\left(g_{n n}^{\mathrm{e}}\right)^{2}+2\left(g_{l l}^{\mathrm{e}}\right)^{2}\right]
\end{aligned}
$$

Care is necessary in defining the principal axes of the $\boldsymbol{g}_{\mathrm{e}}$ tensor, as they are determined by the local symmetry of the free-electron spin, and therefore usually differ from the molecular axes that define the orientational order of the mesogen. There can be competition between paramagnetic and diamagnetic contributions to the macroscopic anisotropy, so the alignment of a paramagnetic mesogen in a magnetic field will be determined by the larger of $\Delta \chi^{\text {dia }}$ and $\Delta \chi^{\text {para }}$.

A further contribution to the molecular paramagnetic susceptibility is from magnetic field-induced distortion of the orbital angular momentum; this is known as temperature-independent paramagnetism (TIP), and is the precise magnetic analog of the electronic polarizability that determines the response of a molecule to a high-frequency electric field. The importance of $\kappa_{\alpha \beta}^{\text {TIP }}$ for mesogens is yet to be established. It is only likely to be significant for molecules with low-lying excited electronic states that are connected to the ground state by magnetically allowed 
dipole transitions; such states are also important in the circular dichroism spectra of molecules. Taking account of all contributions to the molecular susceptibility, the components of the macroscopic susceptibility for a uniaxial liquid crystal composed of uniaxial molecules can be written as

$$
\begin{aligned}
& \chi_{\|}=\bar{\chi}+\frac{2 N \Delta \kappa^{\mathrm{dia}} S}{3}+\frac{2 N \Delta \kappa^{\mathrm{TIP}} S}{3}+\frac{2 N \mu_{0} \mu_{\mathrm{B}}^{2} s(s+1) S}{9 k_{\mathrm{B}} T}\left[\left(g_{n n}^{\mathrm{e}}\right)^{2}-\left(g_{l l}^{\mathrm{e}}\right)^{2}\right] \\
& \chi_{\perp}=\bar{\chi}-\frac{N \Delta \kappa^{\mathrm{dia}} S}{3}-\frac{N \Delta \kappa^{\mathrm{TIP}} S}{3}-\frac{N \mu_{0} \mu_{\mathrm{B}}^{2} s(s+1) S}{9 k_{\mathrm{B}} T}\left[\left(g_{n n}^{\mathrm{e}}\right)^{2}-\left(g_{l l}^{\mathrm{e}}\right)^{2}\right]
\end{aligned}
$$

The description of magnetic anisotropy in liquid crystals containing lanthanide mesogens with metals having f-shell electrons is more complex than given above. Firstly, the quantity that determines the electronic magnetic moment is no longer the spin quantum number $s$, but rather the quantum number for the total angular momentum $\mathbf{J}$ which is the vector sum of the spin and orbital angular momenta, such that $\mathbf{J}=\mathbf{L}+\mathbf{S}$. Secondly, the quantum vector sum (spin-orbit coupling) generates a number of substates, the energies of which may be further influenced by local interactions, that is, the crystal (or ligand) field. The energy separation of these states is such that a number may be populated at normal temperatures, and so any physical properties measured will represent a suitably Boltzmann-averaged sum over the accessible states.

The paramagnetic susceptibility of a free lanthanide atom is isotropic, and is given by

$$
\chi_{0}=\frac{N g_{\mathrm{J}}^{2} \mu_{\mathrm{B}}^{2}}{3 k T} J(J+1)
$$

where $g_{\mathrm{J}}$ is the Landé $\mathrm{g}$-factor, which, for an isolated atom, can be written as

$$
\mathrm{g}_{\mathrm{J}}=1+\frac{J(J+1)+S(S+1)-L(L+1)}{2 J(J+1)}
$$

For a lanthanide atom in a liquid crystal environment, the g-factor becomes a tensor, but in general, none of the principal axes of the tensor coincide with the alignment axis for the liquid crystal. Furthermore, in order to obtain an experimental value for the susceptibility, it must be summed over contributing crystal field (CF) states, for which the g-tensors will be different, and indeed have different principal axes. An approximate theory for this was developed by Bleaney [11] in terms of a hightemperature expansion of the susceptibility in inverse powers of the temperature. However, modern computational methods can now base calculations on a more exact formulation of the susceptibility. 
The quantum-mechanical expression for the magnetic susceptibility tensor of a molecule having a number of accessible states is [12]

$$
\begin{aligned}
\chi_{\alpha \beta}= & \frac{N}{\sum_{j} \exp \left(-E_{j} / k_{\mathrm{B}} T\right)} \\
& \times \sum_{i}\left\{\sum_{j} \frac{\left\langle i\left|\mu_{\alpha}\right| j\right\rangle\left\langle j\left|\mu_{\alpha}\right| i\right\rangle\left\langle i\left|\mu_{\beta}\right| i\right\rangle+\left\langle i\left|\mu_{\beta}\right| j\right\rangle\left\langle j\left|\mu_{\alpha}\right| i\right\rangle}{k T}\right\} \times \exp \left(\frac{-E_{i}}{k_{\mathrm{B}} T}\right)
\end{aligned}
$$

where $k_{\mathrm{B}}$ is Boltzmann's constant. This is a Boltzmann weighted sum over the CF states $(j)$. The first term represents the contribution of the magnetic moment of each CF state, while the second term is equivalent to the temperature-independent paramagnetism identified above. This is only important when there are low-lying excited electronic states connected to the ground state by magnetic-dipole-allowed transitions. Despite the apparent complexity of the problem, calculations have been performed that seem to give quite good agreement with experimental observations. These are discussed below with the results for paramagnetic liquid crystals.

\section{4}

\section{Magnetic Field Effects in Liquid Crystals}

Since intermolecular forces scarcely affect the magnetic susceptibility, measurements of the magnetic anisotropy can provide a direct measure of the orientational order. The anisotropy of susceptibility for a uniaxial liquid crystal phase formed from uniaxial mesogens can be written as

$$
\Delta \chi=N \Delta \kappa S
$$

where, from Equation 2.13, $\Delta \kappa$ contains contributions from diamagnetic, paramagnetic (weak spin-orbit coupling), and TIP terms

$$
\Delta \kappa=\Delta \kappa^{\mathrm{dia}}+\Delta \kappa^{\mathrm{TIP}}+\frac{N \mu_{0} \mu_{\mathrm{B}}^{2} s(s+1)}{3 k_{\mathrm{B}} T}\left[\left(\mathrm{~g}_{n n}^{\mathrm{e}}\right)^{2}-\left(\mathrm{g}_{l l}^{\mathrm{e}}\right)^{2}\right]
$$

$\Delta \kappa$ can be obtained from measurements on single crystals, and provided that accurate values are available for the density, the order parameter $S$ can be obtained directly. An alternative way to determine $S$ from measurements of the temperature dependence of the susceptibility is to fit values to a functional form for the variation of $S$ with temperature. The effects of local biaxial ordering on the measured susceptibility for cyanobiphenyls have been considered by Bunning et al. [9] using crystal data for biphenyl.

Magnetic properties are of importance in the NMR of liquid crystals [13, 14], but the moments of the nuclear spins responsible for the NMR signal are far 
too small to make any contribution to magnetic susceptibilities. However, bulk susceptibility corrections to the NMR chemical shift of a standard immersed in the sample can be used to determine diamagnetic susceptibilities. The chemical shift of the standard is shifted to lower fields in a cylindrical sample owing to bulk magnetization, according to

$$
\sigma_{\text {observed }}=\sigma_{\text {standard }}-\frac{2 \pi}{3} \chi_{i i}
$$

where $\chi_{i i}$ is the susceptibility component in the direction of the external magnetic field. Diamagnetic liquid crystals will align such that the smallest component of $\chi$ is along the magnetic field direction, and this causes a splitting in the NMR lines, which can be related to the order parameter. This technique is extremely useful for obtaining detailed information on the ordering of different segments of flexible molecules $[13,14]$ and can also yield values for the local biaxial order parameters of molecules [13]. The method has been successfully applied to both pure liquid crystals and to dopant molecules dissolved in liquid crystal hosts, which orient the solute molecules. For these experiments, the direction of alignment of the director with respect to the magnetic field is important, and as most liquid crystals have a positive susceptibility anisotropy, the director will align parallel to the magnetic field.

The standard method for measuring magnetic susceptibilities is to use a Faraday balance, which involves the measurement of the force on a sample in an inhomogeneous magnetic field [15]. Other methods use a Gouy balance [10] or a superconducting quantum interference device (SQUID) magnetometer [16, 17]. All methods measure a single component of the susceptibility - the largest for paramagnetic samples, and the smallest for diamagnetic samples, assuming that the alignment of the sample liquid crystal is not constrained by other forces. In order to obtain the anisotropy, a second measurement is required, and this is usually taken as the mean susceptibility measured in the isotropic phase. Diamagnetic susceptibilities are independent of temperature, and it is reasonable to assume that the mean susceptibility in the liquid crystal phase is the same as in the isotropic phase. Hence, for positive materials, the susceptibility anisotropy is given by

$$
\Delta \chi=\frac{3}{2}\left(\chi_{\|}-\bar{\chi}\right)
$$

For liquid crystals having a negative susceptibility, the anisotropy is

$$
\Delta \chi=3\left(\bar{\chi}-\chi_{\perp}\right)
$$

It is not possible to determine the sign of $\Delta \chi$ from magnetic measurements alone.

The ability of magnetic fields to control the alignment of liquid crystals is widely used, for example, in X-ray structural studies of liquid crystals or for optical measurements on aligned liquid crystal films. An advantage of magnetic fields over electric fields for controlling alignment is that complications due to electrical conduction or electrohydrodynamic effects are not present. Competition between aligning fields has been used to obtain direct measurements of susceptibility anisotropies. The basis of the method can be understood from Equation 2.5. 
A similar equation can be written for the free energy density of a liquid crystal in an electric field, such that

$$
g_{\text {elec }}=g_{0}-\frac{1}{2} \varepsilon_{0} E^{2}\left(\varepsilon_{\perp}+\Delta \varepsilon \cos ^{2} \theta\right)
$$

For balancing torques of the electric and magnetic fields on a liquid crystal, the following relation holds

$$
\mu_{0}^{-1} \Delta \chi B^{2}=\varepsilon_{0} \Delta \varepsilon E^{2}
$$

Thus, by measuring the corresponding fields, and knowing the permittivity anisotropy, it is possible to determine $\Delta \chi$ directly [18].

\section{5}

\section{The Frederiks Effect}

The realigning effects of magnetic fields in liquid crystals have been recorded since the earliest period of physical studies of their properties. Most important in this respect is the Frederiks transition, in which the director or optic axis of an aligned liquid crystal film is realigned by an external magnetic (or electric) field. The phenomenon is in modern times associated with applied voltages (and hence electric fields inside the liquid crystal sample). However, the original experiments of Frederiks and coworkers in the 1920s [2] used magnetic rather than electric fields, and in addition, the mathematics of liquid crystal reorientation in a field is much simplified if the field is magnetic rather than electric. For this reason, we include a discussion of the Frederiks effect here.

In a typical Frederiks experiment [3], a liquid crystal cell with planar boundary conditions is subject to a magnetic field applied perpendicular to the substrate plane. The magnetic field couples to the nematic director in such a way that the magnetic energy is lowered if the director rotates so as to lie perpendicular to the cell plane. The director, which lies completely in the plane of the cell, might be expected to be in an unstable equilibrium. There is no magnetic torque on the director in the planar state, but any orientational perturbation is sufficient to create such a couple. If the magnetic torque were the only torque in the system, the magnetic field would then be sufficient to reorient the director in a direction parallel to the field.

However, in fact, owing to elastic and surface anchoring effects, the field only begins to reorient the liquid crystal director above a threshold field, often known as the Frederiks threshold. At this point, the symmetry of the system is spontaneously broken and the director can tilt toward the energetically favored direction in two different ways. This process can be easily be understood using an energy argument (Figure 2.2). 


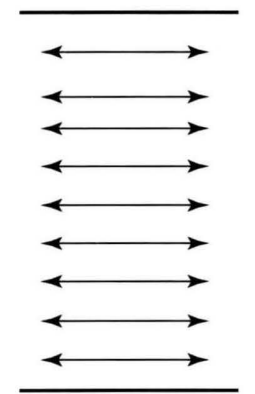

(a)

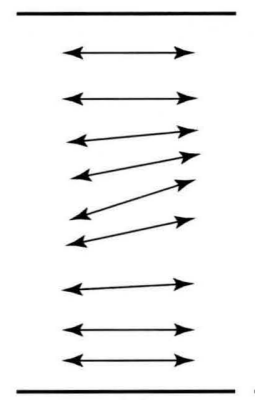

(b)

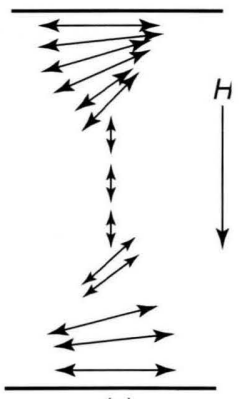

(c)

Figure 2.2 The Frederiks effect in a thin cell. A homogeneous in-plane boundary condition enforces homogeneous orientation inside the cell below the Frederiks threshold field strength. In the figure, the field strength

( $H$ increasing from left to right) favors a direction orientation parallel to the field direction. (a) Below threshold, (b) just above threshold, and (c) well above threshold.

The free energy of the liquid crystal per unit area in a cell of thickness $l(0<z<l)$ can be written as

$$
F=\int_{0}^{l}\left[\frac{1}{2} K\left(\frac{\mathrm{d} \theta}{\mathrm{d} z}\right)^{2}-\frac{1}{2} \Delta \chi B^{2} \sin ^{2} \theta\right] \mathrm{d} z
$$

where we parameterize the reorientation of the director by $\theta(z), K$, is in this case, the splay elastic constant, $\Delta \chi$ is as defined above, and $B$ is the magnetic field. In this case, the boundaries prescribe $\theta(0)=\theta(l)=0$ and, at low fields, this boundary condition is in fact sufficient to constrain $\theta(z)$ everywhere. The Frederiks threshold can be determined by a stability argument as follows:

The boundary conditions require that $\theta(z)$ can always be represented as a Fourier series in $\sin (\pi z / l)$. If the reorientation is small, the first Fourier term will dominate, and

$$
\theta(z) \approx \theta_{0} \sin \left(\frac{\pi z}{l}\right)
$$

where $\theta_{0}$ is the reorientation in the middle of the cell, and can be regarded as a figure of merit for the whole reorientation process. For small $\theta$, a Taylor expansion of $\sin ^{2} \theta$ yields

$$
\sin ^{2} \theta=\theta^{2}-\frac{1}{3} \theta^{4}+\cdots
$$

We can now use

$$
\begin{aligned}
\sin ^{2} \theta(z) & =\theta_{0}{ }^{2} \sin ^{2}\left(\frac{\pi z}{l}\right)-\frac{1}{3} \theta_{0}{ }^{4} \sin ^{4}\left(\frac{\pi z}{l}\right)+\cdots ; \quad\left(\frac{\mathrm{d} \theta(z)}{\mathrm{d} z}\right)^{2} \\
& =\left(\frac{\pi}{l}\right)^{2} \theta_{0}{ }^{2} \cos ^{2}\left(\frac{\pi z}{l}\right)
\end{aligned}
$$


Substituting these results into the free energy expression, and doing the trigonometric integrals, now yields

$$
F \sim\left(K \frac{\pi^{2}}{l^{2}}-\Delta \chi B^{2}\right) \theta_{0}^{2}+\frac{1}{4} \Delta \chi B^{2} \theta_{0}^{4}+\cdots
$$

where the constant of proportionality is unimportant in the present consideration.

This expression shows that the magnetic realignment energy competes with the elastic energy required to turn the director away from its prescribed value at the boundary. For $B<B_{c}=(l / \pi) \sqrt{K / \Delta \chi}$, the energy cost of reorientation is too high. Even for $B$ larger than its threshold value $B_{c}$, the reorientation is far from complete, and $\theta_{0} \sim \sqrt{B^{2}-B_{c}^{2}}$.

The reorientation has an immediate optical signature. It affects birefringence, which induces a change in the phase between the ordinary and extraordinary rays, thus changing the overall polarization, and hence the light transmitted between crossed polarizers. The effect (albeit often when the imposed fields are electric rather than magnetic) is thus used as a method to determine nematic elastic constants. On a more profound level, such threshold effects provide the underlying physics for digital liquid crystal display devices, in particular, the widely used twisted nematic device. In such devices, light is either transmitted or not, corresponding to low or high imposed voltages. In practice, in engineering devices, there are all sorts of complications that make the physics more complicated in detail, but which do not obscure the basic effect.

\section{6 \\ Diamagnetic Liquid Crystals}

Most liquid crystals are diamagnetic and their magnetic anisotropy arises from the electronic structure of the mesogens. Delocalization of electronic charge will enhance the diamagnetic susceptibility, and aromatic groups in particular make a large contribution to the diamagnetic susceptibility. In Table 2.1 are listed molecular susceptibility components for a number of molecules to indicate the likely contributions of various groups to mesogenic structures. These values have been obtained from susceptibility measurements on single crystals.

Most thermotropic mesogens contain aromatic groups, and as the component of the diamagnetic susceptibility perpendicular to a benzene ring is greater than the in-plane component, liquid crystals composed of calamitic mesogens will have a positive diamagnetic anisotropy, while liquid crystals of disklike molecules will have a negative diamagnetic anisotropy. Thus calamitic nematics and smectics will tend to align with their directors along the direction of an external magnetic field, while discotic liquid crystals will align with the director perpendicular to the field. Replacement of aromatic rings by saturated groups such as cyclohexyl, bicyclooctyl, or alkyl chains will reduce the anisotropy of the molecular core, so that liquid crystals based on the trans-trans-cyclohexyl-cyclohexyl core have a negative anisotropy due to the attached terminal groups. Some results for the magnetic 
Table 2.1 Molecular susceptibility components from single crystal measurements.

$\begin{gathered}\kappa_{l} \\ \left(10^{-9} \mathrm{~m}^{3} \mathrm{~mol}^{-1}\right)\end{gathered}$
$\begin{gathered}\kappa_{m} \\ \left(10^{-9} \mathrm{~m}^{3} \mathrm{~mol}^{-1}\right)\end{gathered}$
$\begin{aligned} & \kappa_{n} \\ & \left(10^{-9} \mathrm{~m}^{3} \mathrm{~mol}^{-1}\right)\end{aligned}$

susceptibilities of liquid crystals are given in Table 2.2; more extensive results are given in Chapter 7 by R. Stannarius included in this Handbook (Part II, Volume 3).

\section{7}

\section{Paramagnetic Liquid Crystals}

A large number of paramagnetic compounds that form liquid crystals have been reported in the literature, but their potential for applications has yet to be realized. Magnetic properties have been studied via susceptibility measurements and, in a few cases, using electron paramagnetic resonance, although this technique remains outside the scope of this chapter.

Susceptibility measurements do not usually reveal the sign of the magnetic anisotropy, as only a single component of the susceptibility tensor is measured. Other experiments are necessary such as small-angle X-ray scattering [20] or magnetic birefringence [21-23]. In the latter experiment, the magnetic fieldinduced birefringence (Cotton-Mouton effect) is measured in solutions of the liquid crystal material. The sign of the Cotton-Mouton constant so measured is dependent on the product of the molecular optical anisotropy and the magnetic 
Table 2.2 Diamagnetic susceptibilities of liquid crystals.

\begin{tabular}{llll}
\hline Compound and acronym & $\begin{array}{l}\Delta \chi \\
\left(10^{-9} \mathbf{m}^{3} \mathbf{k g}^{-1}\right)\end{array}$ & $\begin{array}{l}\chi \\
\left(10^{-9} \mathrm{~m}^{3} \mathbf{k g}^{-1}\right)\end{array}$ & References \\
\hline$-C N$ & 0.120 & 0.671
\end{tabular}

$7 \mathrm{CB}$<smiles>N#Cc1ccc(C2CCC([SbH2])CC2)cc1</smiles>

$\mathrm{PCH} 5$<smiles>CCCCCCC</smiles>

$\mathrm{PCH} 7$<smiles>CCCCCCCCC1CCC(C#N)CC1</smiles>

susceptibility anisotropy. Most paramagnetic liquid crystal materials are formed from metal-containing mesogens, for which the optical anisotropy is determined by the ligands; hence, it is usually possible to determine at least the sign of the optical anisotropy independently, thus revealing the sign of the molecular magnetic anisotropy.

Known paramagnetic liquid crystals have a variety of metal centers and coordination geometries $[24,25]$. A requirement for paramagnetism is an unpaired electron spin, but to have an influence on the magnetic anisotropy, there must also be coupling between the spin angular momentum and the orbital angular momentum. For common metals with unpaired d-electrons, spin-orbit coupling is quite weak, and its effects can be formulated in terms of a g-tensor anisotropy. The situation with metals such as lanthanides having unpaired 4 f electrons is more complicated. As explained above, the electronic structure of such metals allows a strong coupling between the electron spin and orbital angular momentum, and the effect on magnetic anisotropy can be large. It is therefore appropriate to consider these two groups of paramagnetic liquid crystals separately. 
Paramagnetic materials may become ferromagnetic as the temperature is lowered (Curie-Weiss behavior). This occurs as the individual magnetic moments become fully aligned in a parallel or antiparallel (antiferromagnetism) arrangement. The identification of paramagnetic liquid crystals raises a question concerning the existence of ferromagnetic liquid crystals, although cooling liquid crystals usually induces crystallization or the formation of glasses.

While ferronematic liquid crystals can be formed by incorporation of magnetic nanoparticles (see below), the formation of a ferromagnetic liquid crystal by intercalation of an Fe(III) complex has been claimed [6]. The dissolution of small amounts of $\mathrm{Fe}(\mathrm{III})$-phthalocyanine into a diamagnetic host discotic liquid crystal 2,9(10), 16(17), 23(24)-tetra(2-decyltetradecyloxy)phthalocyanine resulted in a material, still liquid crystalline, that exhibited hysteresis as measured by SQUID magnetization studies over a wide range of temperatures.

\subsection{1}

\section{Paramagnetic Liquid Crystals Formed by d-Electron Metals}

The effect of competition between diamagnetic and paramagnetic contributions to the susceptibility is illustrated by the behavior of salicylaldimine complexes of copper [26, 27]. These are formed from copper(II) having a d9 electron configuration, which results in a square planar geometry around the metal center. The g-tensor anisotropy is such that $\left(\mathrm{g}_{n n}^{\mathrm{e}}\right)^{2}<(1 / 2)\left(\left(\mathrm{g}_{l l}^{\mathrm{e}}\right)^{2}+\left(\mathrm{g}_{m m}^{\mathrm{e}}\right)^{2}\right)$ so that the paramagnetic contribution to the anisotropy is negative. For complexes with four benzene rings in the structure (Table 2.3, compounds 1 and 2), the paramagnetic term is larger

Table 2.3 Magnetic properties of salicylaldimine complexes of copper(II) [26, 27].

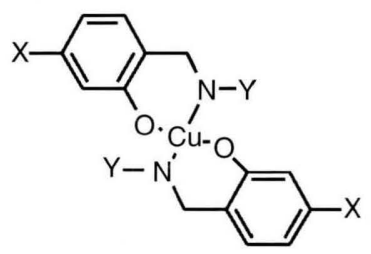

\begin{tabular}{llll}
\hline$Y$ & $\Delta \chi^{\text {para }}$ & $\Delta \chi^{\text {dia }}$ & $\begin{array}{c}\text { Orientation } \\
\text { to magnetic } \\
\text { field }\end{array}$ \\
& $\left(10^{-9} \mathrm{~m}^{3} \mathrm{~mol}^{-1}\right)$ & $\left(10^{-9} \mathrm{~m}^{3} \mathrm{~mol}^{-1}\right)$ &
\end{tabular}

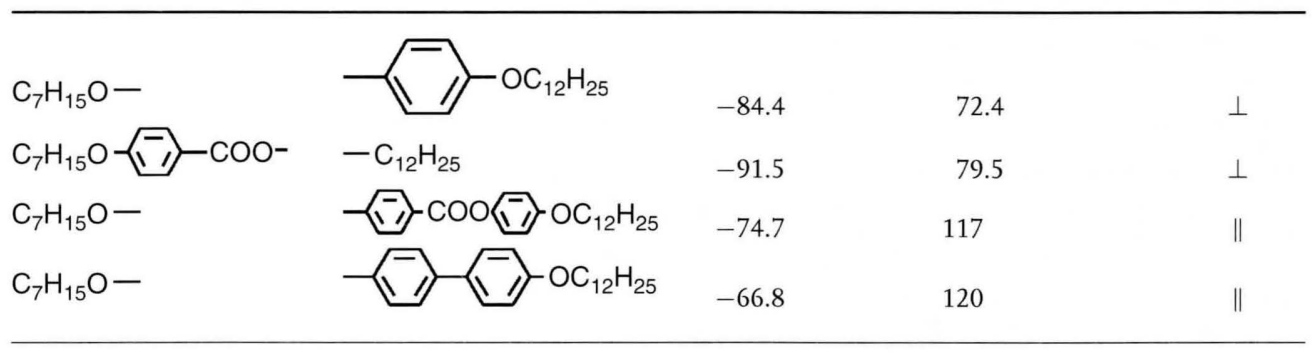


than the diamagnetic term in the anisotropy, and so the complexes align with the major axis $(n)$ perpendicular to the field direction - free rotation about the long molecular axis is assumed. Increasing the number of benzene rings to six (Table 2.3 compounds 3 and 4) causes the diamagnetic anisotropy to dominate, and the director aligns parallel to a magnetic field (Figure 2.3).

Electron paramagnetic resonance measurements on these liquid crystals give $g$-values of $\mathrm{g}_{n n}^{\mathrm{e}}=2.053$ and $1 / 2\left(\mathrm{~g}_{l l}^{\mathrm{e}}+\mathrm{g}_{m m}^{\mathrm{e}}\right)=2.082$. By contrast, the corresponding vanadyl (VO) $\mathrm{d}^{1}$ complexes have a reversed g-tensor anisotropy $\mathrm{g}_{n n}^{\mathrm{e}}=1.987$ and $1 / 2\left(g_{l l}^{\mathrm{e}}+\mathrm{g}_{m m}^{\mathrm{e}}\right)=1.966$, and so these complexes always align with their long molecular axes along the magnetic field direction.

\subsection{2}

\section{Paramagnetic Liquid Crystals Formed by f-Electron Metals: the Lanthanides}

The lanthanide elements (lanthanum La, cerium Ce, praseodymium Pr, neodymium Nd, promethium Pm, samarium Sm, europium Eu, gadolinium Gd, terbium Tb, dysprosium Dy, holmium Ho, erbium Er, thulium Tm, ytterbium $\mathrm{Yb}$, and lutetium $\mathrm{Lu}$ ) have atomic numbers 57-71 and their electronic structure corresponds to the filling of the $4 \mathrm{f}$-shell from $\mathrm{La}\left(4 \mathrm{f}^{0}\right)$ to $\mathrm{Lu}\left(4 \mathrm{f}^{14}\right)$. The particular properties of $4 \mathrm{f}$-electrons confer special chemical and physical properties on the lanthanide atoms, ions, and their compounds. From a chemical point of view, the lanthanide elements can support high coordination numbers, that is, it is possible to create compounds with a relatively large number of ligands (often up to eight), and this provides much scope for inventive chemistry. For electronic physical properties, the special features of f-electrons results in closely spaced electronic states and interesting luminescent properties; furthermore, states having high electron spins can have unusual magnetic properties. Both these properties can be important for liquid crystals.

Liquid crystals containing lanthanide elements have been prepared and they form a subset of so-called metal-containing liquid crystals. Their preparation and properties have been pursued by a few research groups and a range of liquid crystals have been prepared with a variety of interesting physical properties. The first such materials were prepared by the group in Kazan in 1991 [28] and subsequently a large number of other liquid crystals have been prepared [29]. In this section, we wish to focus on the paramagnetic properties of these materials.

The great interest in and potential for lanthanide-containing liquid crystals is their high magnetic anisotropy [30]. This has been measured for a number of materials and is found to be two or three orders of magnitude higher than for other paramagnetic liquid crystals. The origin of the high anisotropy is the strong spin-orbit coupling exhibited by the $4 \mathrm{f}$-electrons of the lanthanide atoms or ions. These have been calculated [12] using the Gerloch-McMeeking formula (Equation 2.16 above) and are in only approximate agreement with experimental results. However an interesting result from the calculations, confirmed by experiment, is that depending on the lanthanide element, the sign of the magnetic anisotropy may 

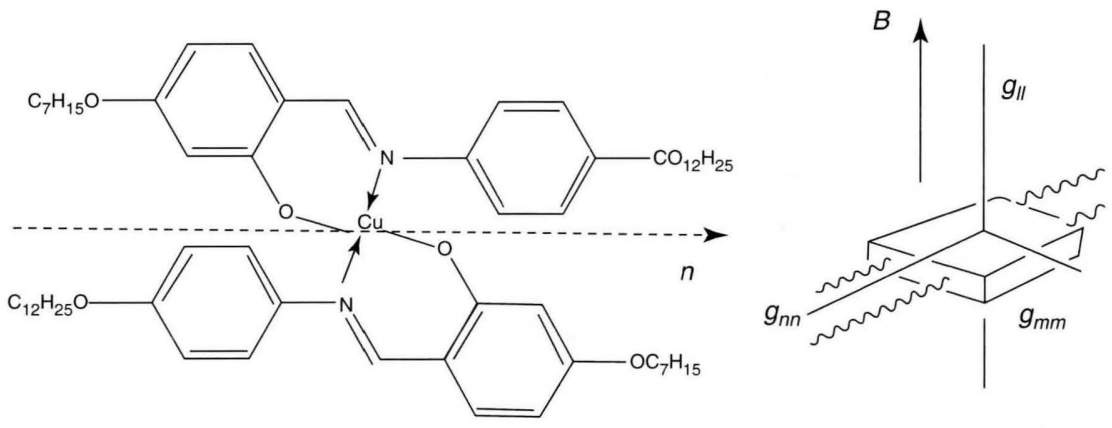

(a)

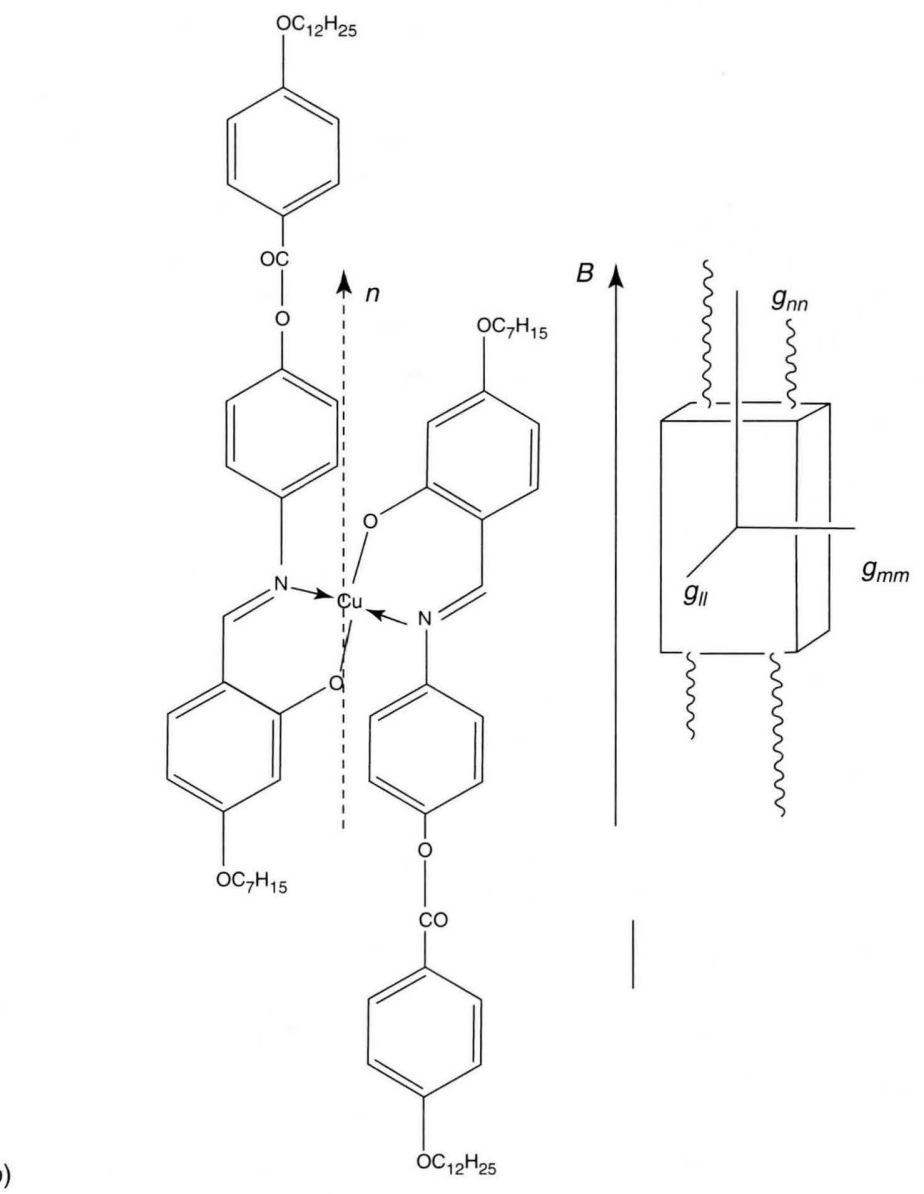

(b)

Figure 2.3 Alignment of salicylaldimine complexes in a magnetic field. (a) $\Delta \chi-v e, \mathbf{n}$ perpendicular to the field and (b) $\Delta \chi+v e, \mathbf{n}$ parallel to the field. 
be positive or negative. The two groups of elements in oxidation state III are as follows with opposite signs for their magnetic anisotropy: Ce(III), $\operatorname{Pr}(\mathrm{III}), \mathrm{Nd}(\mathrm{III})$, $\mathrm{Sm}(\mathrm{III}), \mathrm{Tb}(\mathrm{III}), \mathrm{Dy}(\mathrm{III})$, and $\mathrm{Ho}(\mathrm{III})$ in the first group and $\mathrm{Eu}(\mathrm{III}), \mathrm{Er}(\mathrm{III}), \mathrm{TM}(\mathrm{III})$, and $\mathrm{Yb}(\mathrm{III})$ in the second. Which group has a negative magnetic anisotropy and which group has a positive magnetic anisotropy depends on the symmetry of the CF acting on the lanthanide ion [31]. Thus different coordination geometries give rise to different anisotropies, but the different lanthanide-containing materials always share the same sign of anisotropy as others in their group.

The nature of the chemical structures for lanthanide-containing mesogens is that smectic phases A are formed. The complexes are often ionic materials, so the counter anions will also influence the liquid crystal properties. Lanthanide soaps formed from the ions and fatty acids also form liquid crystalline lamellar structures, although their magnetic properties have not been extensively investigated [32]. An example of the magnetic behavior of a lanthanide Schiff's base complex is given in Figure 2.4 [33]. Results for the magnetic anisotropy of a number of different

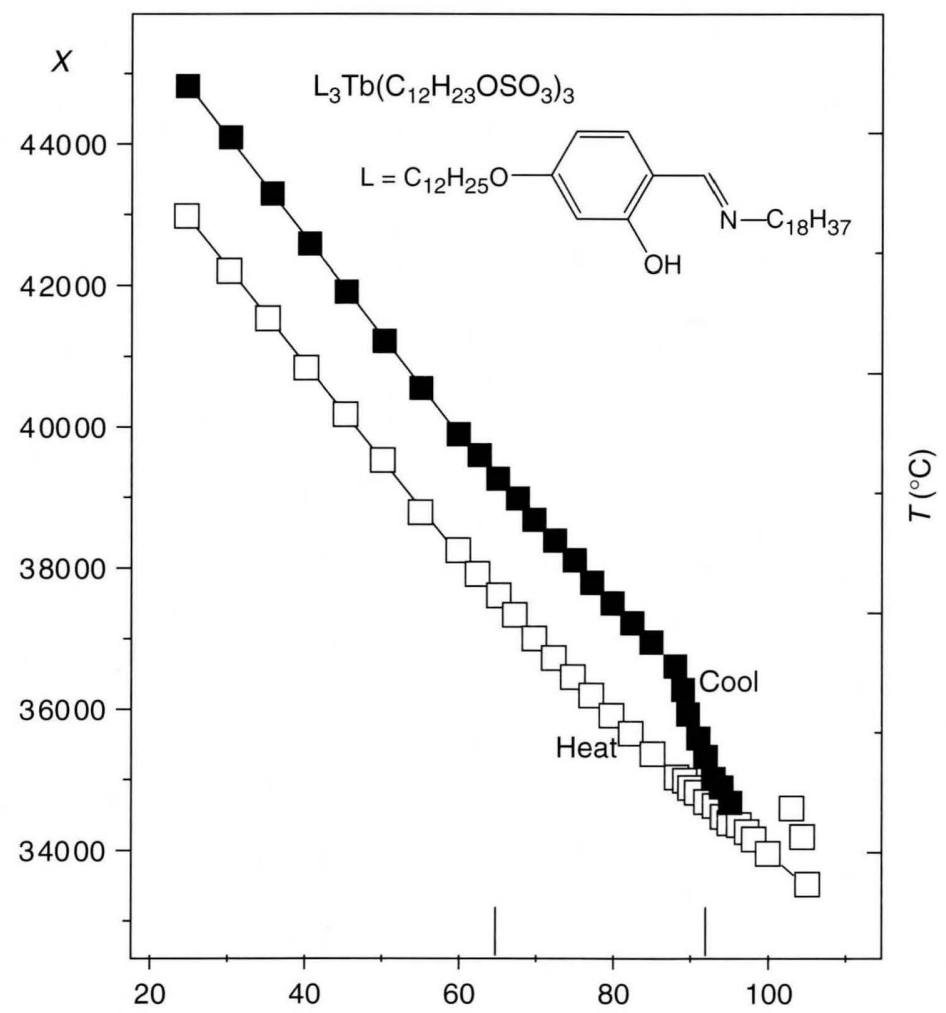

Figure 2.4 Temperature dependence of the molar susceptibility (in units of $\left.10^{-6} \mathrm{~cm}^{3} \mathrm{~mol}^{-1}\right)$ for the rare earth ionic mesogen $\mathrm{L}_{3} \mathrm{~Tb}\left(\mathrm{C}_{12} \mathrm{H}_{23} \mathrm{OSO}_{3}\right)_{3}$ : heating $(\square)$ and cooling (ם). The transition from isotropic to smectic $A$ appears on cooling at $92^{\circ} \mathrm{C}$. (Source: Taken from Ref. [33].) 


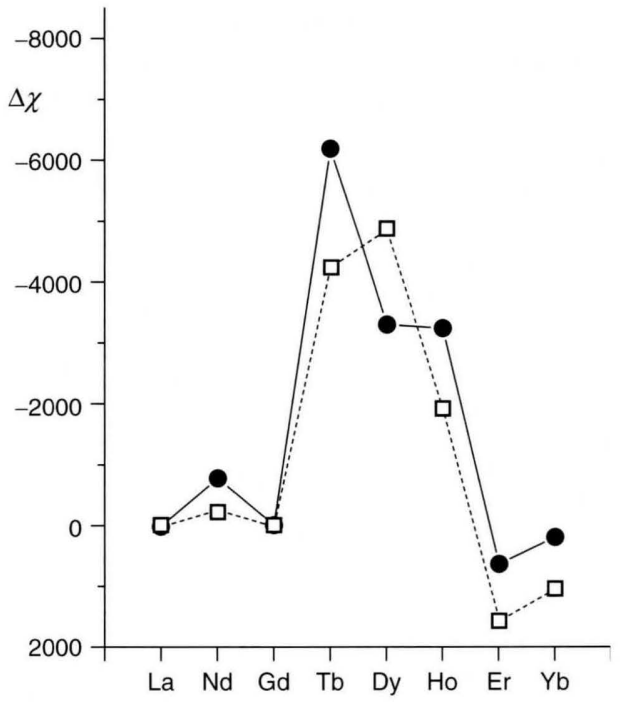

Figure 2.5 Magnetic anisotropies of the smectic A phase of ionic lanthanide (Ln) mesogens $\mathrm{L}_{3} \mathrm{Ln}\left(\mathrm{C}_{12} \mathrm{H}_{23} \mathrm{OSO}_{3}\right)_{3}$. Experimental values $(\bullet)$ are compared with theoretical estimates $(\square)$ based on the crystal field model for paramagnetic susceptibility. (Source: Taken from Ref. [33].)

lanthanide complexes are given in Figure 2.5 [33], where they are compared with values calculated using the approximate theory referred to above.

An alternative approach to the preparation of lanthanide-containing liquid crystals has been to dissolve lanthanide salts in a liquid crystal host. In particular, up to $3 \mathrm{~mol} \%$ of a selection of lanthanide bromides $\left(\mathrm{EuBr}_{2}, \mathrm{SmBr}_{3}, \mathrm{TbBr}_{3}\right.$, and $\left.\mathrm{DyBr}_{3}\right)$ have been dissolved in the smectic A phase of the ionic liquid crystal 1-dodecyl-3methylimidazolium bromide. The photoluminescence of these mixtures have been examined [34], but not their magnetic properties.

A characteristic of ionic liquid crystals is a tendency to form smectic A phases; such phases are difficult to align and have a high viscosity. However, nonionic lanthanide complexes exhibiting both nematic and smectic phases have been prepared [35], and these are expected to have lower viscosities in the nematic phases. The structure and properties of these complexes are given in Table 2.4.

In spite of the considerable success in synthesizing metallomesogens with very large magnetic anisotropies, they have not so far been exploited in devices. The presence of metal ions increases the conductivity considerably and therefore tends to exclude their use in applications that rely on both magnetic and electric fields. Because of their molecular structure, the metal-containing magnetic liquid crystals have relatively high viscosities, which limit the speed of response in dynamic applications. Despite such relatively large magnetic susceptibilities, strong magnetic fields are still required to drive the reorientation. 
Table 2.4 Mesophase transition temperatures and magnetic susceptibility anisotropy for lanthanide tris- $\beta$-diketonate-bipyridyl complexes [23].

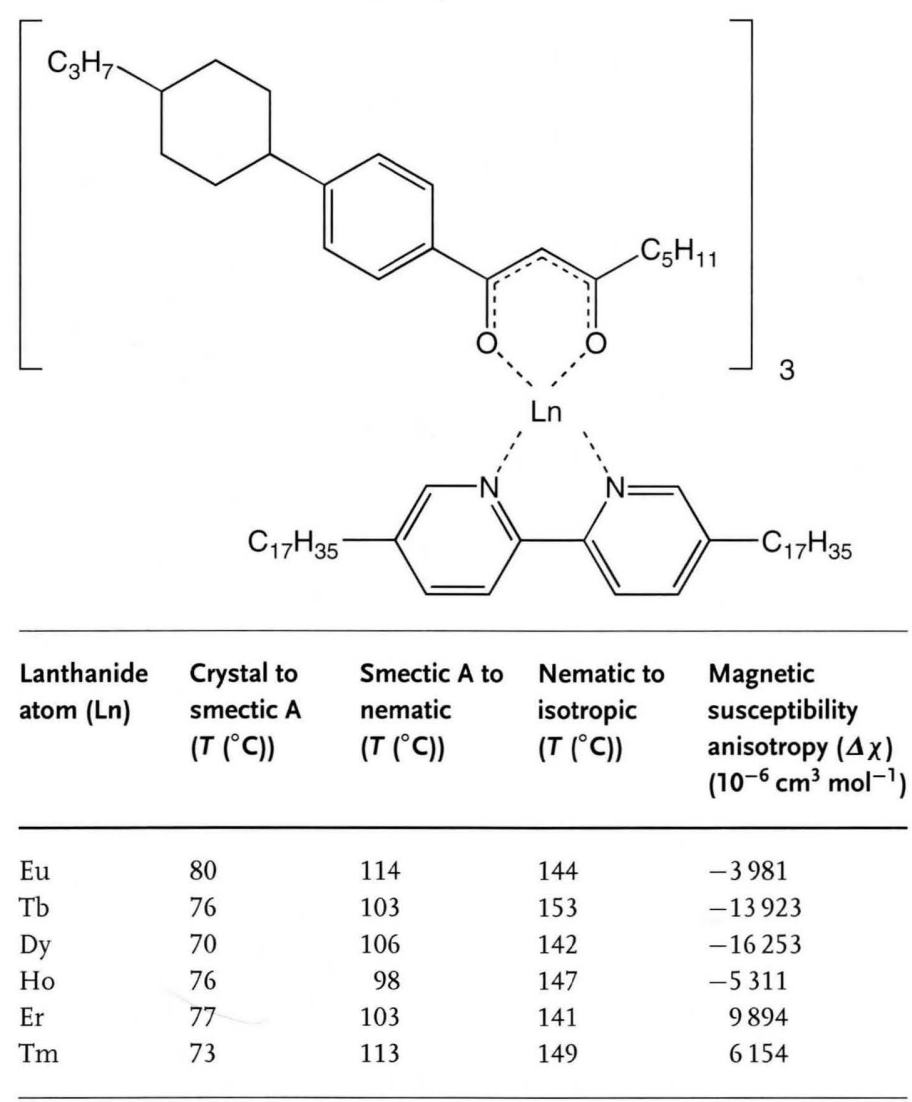

\section{8}

\section{Ferromagnetism and Related Phenomena in Liquid Crystals}

\subsection{1}

\section{Ferromagnetism}

At sufficiently low reduced temperatures, and/or strong spin-spin interactions, spin magnetic moments can become ordered in a parallel array to give ferromagnetic materials, or ordered in an antiparallel fashion to give an antiferromagnetic structure. This behavior is rare in organic materials, for which ferromagnetic organization only occurs at low temperatures. There is a requirement for unpaired electron spins and so in the context of liquid crystals, possible ferromagnetic materials will almost certainly require metal-containing mesogens. The contribution of ferromagnetic or antiferromagnetic coupling to the susceptibility can be described in terms of a modified Curie law, known as the Curie-Weiss law, and the isotropic 
susceptibility can be written as

$$
\bar{\chi}^{\text {para }}=\frac{N \mu_{0} g_{\mathrm{e}}^{2} \mu_{\mathrm{B}}^{2} s(s+1)}{3 k_{\mathrm{B}}(T-\Theta)}
$$

where the characteristic temperature $\Theta$, the Curie temperature, is a measure of the ferromagnetic coupling, and marks the onset of permanent magnetization. If $\Theta$ is negative, then the local magnetic interactions are antiferromagnetic and $\Theta$ is called the Neel temperature.

The possibility for a mesogenic material exhibiting ferromagnetism was long considered remote. In the context of the experimental search for ferromagnetic liquid crystals, Kats and Lebedev [36] made a theoretical study of possible properties of ferromagnetic liquid crystals. They predicted that magnetic spin waves and nematic hydrodynamic modes would interact. This would imply changes in the effective nematic viscosity, with possible device implications.

On the experimental side, organic ferromagnets have been prepared [37, 38], but they mostly have very low Curie temperatures, well below the melting points of the compounds. Since the origin of ferromagnetism lies in long-range spin-spin interactions, these were thought unlikely to persist in a fluid liquid crystalline state. A more fruitful route to ferromagnetic ordering may lie in metal-containing liquid crystal polymers [39]. Ferromagnetic response at room temperature has indeed been observed more recently in guest-host discotic liquid crystals that include paramagnetic iron [6].

\subsection{2}

\section{Ferronematics}

Despite the single report so far of a ferromagnetic liquid crystal [6], the phenomenon of true spontaneous ferromagnetism in liquid crystals seems at the moment to be rare. An alternative strategy toward creating magnetic materials with liquid crystalline properties is to synthesize liquid crystalline ferrofluids. Ferrofluids are colloidal suspensions of single-domain magnetic particles in a liquid carrier and they possess extensive technological applications [40, 41]. Examples are colloidal suspensions of ferrite $\gamma-\mathrm{Fe}_{2} \mathrm{O}_{3}$, magnetite $\mathrm{Fe}_{3} \mathrm{O}_{4}$, or cobalt metal in either hydrocarbon or water-based solvents.

The idea of using such systems in a liquid crystalline context is due to Brochard and de Gennes [7], who labeled such systems ferronematics. Their idea was that orientational coupling between the magnetic colloidal particles and the nematic director would dramatically increase the effective director-magnetic field interaction. A consequence would be the possibility of manipulating nematic properties with low magnetic fields. In particular, the Frederiks transition in a typical liquid crystal cell (thickness $10-100 \mu \mathrm{m}$ ) would now be on the scale of tens of gauss, rather than of thousands.

Important conditions for ferronematics, which we discuss in more detail below, are as follows:

1) The colloidal volume concentration should be very low. 
2) The colloidal dimension should be in the nanocolloid regime ( $\leq 10 \mathrm{~nm})$.

3) The response of the colloidal particles to a magnetic field should be collective rather than individual.

4) No significant aggregation of colloidal particles should take place.

5) The resulting colloidal suspensions should be stable.

Theoretical estimates suggest that under favorable circumstances, concentrations of even one part in $1000\left(10^{-4}\right.$ by volume) might be sufficient to exhibit the giant magnetic susceptibilities. At sufficiently low colloidal concentrations, the essential liquid crystal properties would be retained. If colloidal concentrations are too high (or the colloidal particles are too large), then a rich menagerie of defect structures can form around the colloidal particles. The defects disrupt the uniform nematic director structure and also scatter light strongly [42]; consequently, these systems are of less interest in an optoelectronic device context. If the volume concentration is too low, then the colloidal particles might be expected to respond to external fields independently rather than collectively. Burylov and Raikher [43] have estimated that a minimum critical volume concentration $f^{*} \sim 10^{-6}$ would be required in order to establish this collective behavior. Finally, we note that aggregation of ferroparticles decreases the likelihood of collective behavior. In addition, the aggregates scatter light significantly, with adverse device implications. In practice, this aggregation can be difficult to avoid unless sophisticated surfactants are used to coat the individual colloidal particles. Indeed, the aggregation can be such as to cause the suspensions to be unstable and to phase separate into separate colloid-rich and colloid-poor systems. A review of some aspects of ferronematics in a more general ferrofluid context has been given by Scherer and Figueiredo Neto [44].

\subsubsection{Theory}

Following the work of Brochard and de Gennes [7], a detailed theory of ferronematics was developed by Burylov and Raikher [45] (see also the review by Raikher [46]), and further discussed by Zadorozhnii et al. [47]. The free energy density can be written in terms of the local director, $\mathbf{n}$, and the magnetic director, $\mathbf{m}$, denoting the direction of the local magnetic polarization. It is then a sum of the usual Oseen-Frank elastic terms, together with the usual quadratic term coupling the director with the magnetic field, an extra linear term coupling the magnetic director with the magnetic field, and a quadratic term coupling the magnetic and nematic directors. This then yields [43, 45-47]

$$
F=\int_{v} \mathrm{~d} V\left\{\begin{array}{c}
\frac{1}{2}\left[K_{1}(\nabla \cdot \mathbf{n})^{2}+K_{2}(\mathbf{n} \cdot \nabla \times \mathbf{n})^{2}+K_{3}(\mathbf{n} \times \nabla \times \mathbf{n})^{2}\right] \\
-\frac{1}{2} \chi_{\mathrm{a}}(\mathbf{n} \cdot \mathbf{H})^{2}-f \bar{M}(\mathbf{m} \cdot \mathbf{H})-f W_{\mathrm{p}}(\mathbf{n} \cdot \mathbf{m})^{2}
\end{array}\right\}
$$

where the $K_{i}$ are the Oseen-Frank elastic constants, $\chi_{\mathrm{a}}$ takes its usual meaning (as described above), $\bar{M}$ is the mean magnetic moment per colloidal particle, $f$ is the volume concentration of magnetic colloid particles, and $W_{\mathrm{p}}$ is a coupling constant describing the anchoring per colloidal particle. In the example here, it is favorable for $\mathbf{n}$ and $\mathbf{m}$ to be parallel, $W_{\mathrm{p}}$ is positive, and the magnetic particles are locally uniformly aligned. However, the theory is not changed qualitatively in 
cases when the particle geometry favors a perpendicular configuration or when the magnetic particles are incompletely aligned. In the latter case, it is also necessary to introduce a local dipolar magnetic order parameter $p$. It is a task for microscopic theory to calculate $W_{p}$ (see, for example Burylov and Raikher [45]). In the mesoscopic theory, however, the details of this calculation do not enter. More sophisticated theories allow the concentration $f$ to change as a function of position, subject only to a chemical potential condition, and include the entropy of solution.

Often experiments include both a field in the plane of the cell, as well as a field perpendicular to it. The planar field is known as a bias field, and its purpose is to align the magnetic moments in the plane, so that the perpendicular field can then rotate the moments toward the normal direction. A consequence is that the low field rotation of the director becomes linear in the perpendicular field. Without the bias field, the magnetic liquid crystal colloid is said to be compensated; the net average magnetic dipole is zero. In such a case, the threshold Frederiks phenomenon is restored. An interesting prediction [47], so far unobserved, involves the possibility of an inverse Frederiks transition at high fields, at which the nematic director unbinds from the magnetic director and returns to an undistorted state.

\subsubsection{Experimental Observations: Thermotropic Liquid Crystals}

The first experimental demonstration of ferronematic behavior in thermotropic liquid crystals is due to Rault et al. [48], who studied colloidal suspensions of maghemite $\left(\gamma-\mathrm{Fe}_{2} \mathrm{O}_{3}\right)$ rods $(L / D \sim 9)$ in MBBA. The rods had an aspect ratio of about 9 , with colloid volume fraction $f \sim 5 \times 10^{-3}$. Hysteresis was observed below the nematic-isotropic phase transition. Interestingly, in high fields, chaining of the magnetic particles was observed. Hayes [49] prepared a sample of magnetic $\mathrm{Fe}_{3} \mathrm{O}_{4}$ platelets and observed the platelets rotating in a magnetic field. In neither case, however, were Frederiks experiments carried out to examine changes in effective magnetic anisotropic interaction. Such an experiment was carried out by Chen and Amer [50]. These authors observed the low field linear distortion discussed above, as well as a set of textures indicating orientational distortion at magnetic fields of only a few Gauss. But it seems likely that nanoparticle aggregation occurred; the lack of long-term stability and uniformity in ferromagnetic samples remains a major challenge.

Further extensive investigations of ferronematics, such as those carried out by Kopčanský and coworkers [51], have explored different thermotropic liquid crystals as a base material and nanoparticles of different shapes and sizes. The principal experimental method has been to use capacitance as a signal of reorientation in a magnetic field. The presence of ferromagnetic nanoparticles in a liquid crystal suspension may, but does not always, yield a lower magnetic Frederiks threshold. The same team has also found noticeable increases in the nematic-isotropic phase transition temperature in magnetic liquid crystal colloids with respect to pure materials [52].

Other workers [53-55] have used optical methods to probe the Frederiks transition in ferronematics. In general, the magnetic Frederiks threshold field is reduced 
by the presence of the magnetic field. But the magnitude of the reduction is of the order of $30 \%$, rather than the large factor suggested theoretically. We note, however, that there are strong effects at very low fields [55].

In conclusion, generally speaking, it is possible to fit the experimental results such as Refs [51-55] with the phenomenological theories of the type of Refs [43, 45-47]. Thus, although unambiguous ferronematic effects have been observed in thermotropic liquid crystals, it remains an open question whether it is possible to create low magnetic particle volume concentration thermotropic ferronematics with the giant response originally envisaged by Brochard and de Gennes in their seminal 1970 paper.

\subsubsection{Experimental Observations: Other Liquid Crystalline Systems}

The doping of lyotropic liquid crystals with water-based surfacted ferrofluids was achieved in 1979 by Liebert and Martinet [56]. Matuo et al. [57] studied a lyotropic ferronematic formed by doping with $\mathrm{CoFe}_{2} \mathrm{O}_{4}$, and extended the theory of Brochard and de Gennes [7] to this system. The dynamics of a lyotropic ferronematic were studied by Figueiredo Neto et al. [58]. More recently, Berejnov et al. [59] synthesized colloidal solutions of ferrite nanoparticles in the nematic phase of the lyotropic surfactant micellar system potassium laurate/1-decanol/water. The resulting ferronematic samples were reversibly orientable by low magnetic fields ( $\sim 100$ Oe). Moreover, these samples retained their transparency and stability for several months. In some cases, lyotropic liquid crystalline systems are prepared in which the active orienting elements are themselves magnetic. For example, Lemaire et al. [60] have discussed the magnetic properties of colloidal aqueous suspensions of goethite $(\alpha-\mathrm{FeOOH})$ lath-shaped nanorods. Progress before 2005 on lyotropic ferronematics and related systems has been reviewed by Scherer and Figueiredo Neto [44].

A particular item of interest concerns the preparation of ferromagnetic particles. Walton and Shibli [61] found, for example, that magnetite particles $\left(\mathrm{Fe}_{3} \mathrm{O}_{4}\right)$ were only paramagnetic in isotropic solutions, but became ferromagnetic in a potassium laurate/1-decanol/water ( $\mathrm{KL} / \mathrm{DeOH} / \mathrm{H}_{2} \mathrm{O}$ ) lyotropic liquid crystal solution.

Lyotropic ferrosmectic materials were first prepared by Fabre et al. [62], while a ferrofluid-doped liquid crystalline elastomer has been studied by Figueiredo Neto et al. [58]. Such systems exhibit an enhanced interaction between magnetic field and strain. Finally, we note the possibility that multiferroic colloidal mixtures could play a role in sophisticated liquid crystal devices [63].

\section{Appendix. Units and Conversions}

Any review of magnetic properties must deal with the confusing problem of units. Experimental data is presented in the literature using different systems of units and conventions, and relating measurements quoted using one particular system to others adopting a different convention can be demanding. The problem of units for magnetic properties stems in part from a historical misidentification of variables in Maxwell's equations, and some properties are defined in terms of 
the magnetic field strength $(H)$ and others in terms of the magnetic induction $(B)$. Occasionally, these terms are used interchangeably, but they have different units and dimensions and refer to different quantities. The most difficult aspect of assessing measurements in the literature is that authors quoting the results from others often convert them to different units. As a consequence, different quoted values for magnetic susceptibilities may simply be a result of the different units or conventions used.

In an attempt to resolve this, we review experimental results for the magnetic susceptibility of two key liquid crystalline materials, $p$-azoxyanisole (PAA) and $7 \mathrm{CB}$, from various authors and compare the results and make the conversions from different systems of units. The preferred system of units is Le Système Internationale d'Unités (SI), and the reference used for defining units in this chapter is Quantities, Units and Symbols in Physical Chemistry, Second Edition, International Union of Pure and Applied Chemistry, Physical Chemistry Division, Blackwell Scientific Publications, Oxford 1993.

The so-called volume susceptibility tensor $\chi_{\alpha \beta}$ is defined in terms of the free energy density $\left(\mathrm{J} \mathrm{m}^{-3}\right)$ as

$$
\mathrm{g}=\mathrm{g}_{0}-\frac{1}{2} \mu_{0}^{-1} \chi_{\alpha \beta} B_{\alpha} B_{\beta}
$$

where $B$ is the magnetic induction and $\mu_{0}$ is the magnetic permeability of free space $\left(\mu_{0}=4 \pi \times 10^{-7} \mathrm{~N} \mathrm{~A}^{-2}\right)$. In fact, the susceptibility $\chi_{\alpha \beta}$ so defined is an intensive dimensionless quantity, just like the relative electric permittivity, and so has no units, as can be established from Equation A.1, noting that the unit of $B$ is a tesla $\left(1 \mathrm{~T}=1 \mathrm{~J} \mathrm{~A}^{-1} \mathrm{~m}^{-2}\right)$. The cgs unit of magnetic induction is gauss, and $1 \mathrm{~T}=10^{4} \mathrm{G}$. It is possible to define the magnetic susceptibility in terms of the magnetic field $H$, in which case the free energy density becomes

$$
\mathrm{g}=\mathrm{g}_{0}-\frac{1}{2} \mu_{0} \chi_{\alpha \beta} H_{\alpha} H_{\beta}
$$

with $H_{\alpha}=\mu_{0}{ }^{-1} B_{\alpha}$. The SI unit of magnetic field is amperes per meter, and the definition of $\chi_{\alpha \beta}$ is the same as in Equation A.1. For uniaxial materials, the susceptibility can be expressed in terms of the mean susceptibility $\bar{\chi}=$ $(1 / 3)\left(\chi_{\|}+2 \chi_{\perp}\right)$, where $\chi_{\|}$is the parallel and $\chi_{\perp}$ is the perpendicular component of the susceptibility, and the susceptibility anisotropy is $\Delta \chi$.

$$
\chi_{\alpha \beta}=\bar{\chi} \delta_{\alpha \beta}+\frac{1}{3} \Delta \chi\left(3 n_{\alpha} n_{\beta}-\delta_{\alpha \beta}\right)
$$

The vector $n_{\alpha}$ defines the orientation of the optic axis (director) in the chosen coordinate frame.

In the liquid crystal literature, there is no consistency in the use of units for magnetic susceptibility. Indeed a number of authors have used cgs-emu units, 
which were the fashion before SI became established. The classic text by de Gennes and Prost [64] uses the earlier system of units, and refers to magnetic fields in oersteds. This raises another problem of comparison, which relates to the use of rationalized or irrationalized equations, that is, the appearance of $4 \pi$ in appropriate equations. Thus, if the susceptibility $\chi^{\text {(ir) }}$ is derived from an irrationalized equation, then the susceptibility in a rationalized system is $\chi=4 \pi \chi^{(\mathrm{ir})}$. The magnetic free energy density for a uniaxial liquid crystal can be written as [64]

$$
\mathrm{g}=\mathrm{g}_{0}-\frac{1}{2} \mu_{0}^{\mathrm{cgs}} \chi_{\perp}^{\mathrm{cgs}} H^{2}-\frac{1}{2} \mu_{0}^{\mathrm{cgs}} \Delta \chi^{\mathrm{cgs}} n_{\alpha} H_{\alpha} n_{\beta} H_{\beta}
$$

where the magnetic permeability of free space in cgs units has been included: $\mu_{0}^{\mathrm{cgs}}=1 \mathrm{~cm} \mathrm{~g} \mathrm{~s}^{-2}$ (emu current) ${ }^{-2}$ and 1 emu of current $=10 \mathrm{~A}$. Oersteds are irrational units of magnetic field, and the conversion is $1 \mathrm{Oe}^{(\mathrm{ir})}=10^{3} \mathrm{~A} \mathrm{~m}^{-1}$. Assuming that $\Delta \chi^{\text {cgs }}=10^{-7}$ (a typical value), and for a magnetic field of $10^{4} \mathrm{Oe}$, then the corresponding anisotropic magnetic contribution to the free energy density from Equation A. 2 is $10 \mathrm{erg} \mathrm{cm}^{-3}$, which becomes $1 \mathrm{~J} \mathrm{~m}^{-3}$. It is instructive to do the same calculation for the free energy density using Equation A.1. For $\Delta \chi^{\mathrm{SI}}=10^{-7}$, and a magnetic induction of $1 \mathrm{~T}$, which corresponds to a magnetic field of $10^{4} \mathrm{Oe}$, the corresponding anisotropic magnetic free energy density is $(4 \pi)^{-1} \mathrm{~J} \mathrm{~m}^{-3}$. Then the conversion from cgs units to SI units for the volume susceptibility is

$$
\chi^{\mathrm{SI}}=4 \pi \chi^{\mathrm{cgs}}
$$

The potential confusion over units and the fact that results are often quoted for differently defined susceptibilities, that is, volume susceptibility or mass susceptibility, often makes it difficult to compare data from different sources. Also different techniques to measure magnetic susceptibilities measure different quantities. For example, the Faraday-Curie balance determines the susceptibility along the direction of the magnetic field gradient, that is, $\chi_{\|}$for positive anisotropy materials and the mean susceptibility $\bar{\chi}$ in the isotropic phase, while compensation methods measure the anisotropy directly.

In an attempt to make data on magnetic susceptibilities more accessible, data for two standard materials PAA and 7CB from a number of sources is given in Table 2.A.1, quoting both the primary data and the appropriate conversions to SI units. In converting from volume to mass susceptibilities or molar susceptibilities, a value for the density is required. Sometimes, experimental results are available, but often it is assumed that the density of a nematic liquid crystal is $1 \mathrm{~g} \mathrm{~cm}^{-3}$ or $1 \times 10^{3} \mathrm{~kg} \mathrm{~m}^{-3}$. Furthermore, as the magnetic susceptibility anisotropy is directly proportional to the order parameter, it is dependent on temperature. The relationships between volume, mass, and molar susceptibilities are as follows:

$$
\begin{aligned}
\left.\chi^{\mathrm{m}} \text { (mass susceptibility }\right) \mathrm{m}^{3} \mathrm{~kg}^{-1} & =\chi(\text { volume susceptibility }) \rho^{-1} \\
\left.\chi^{\mathrm{M}} \text { (molar susceptibility }\right) \mathrm{m}^{3} \mathrm{~mol}^{-1} & =\chi^{\mathrm{m}} \times(\text { molecular weight in kilogram })
\end{aligned}
$$


Table 2.A.1 Calibration data for magnetic susceptibilities.

\begin{tabular}{|c|c|c|c|c|c|c|c|c|c|c|}
\hline & \multicolumn{4}{|c|}{ Primary data (with units) } & \multicolumn{5}{|c|}{ Rationalized SI } & \multirow[t]{2}{*}{ References } \\
\hline & Temperature & $\begin{array}{l}\chi_{\|} \\
\text {(type) }\end{array}$ & $\begin{array}{l}\chi_{\text {av }} \\
\text { (type) }\end{array}$ & $\begin{array}{l}\Delta \chi \\
\text { (type) }\end{array}$ & $\begin{array}{l}\chi_{\|} \\
\text {(type) }\end{array}$ & $\begin{array}{l}\chi_{\text {av }} \\
\text { (type) }\end{array}$ & $\begin{array}{l}\Delta \chi \\
\text { (vol) }\end{array}$ & $\begin{array}{l}\Delta \chi \\
\text { (mass) } \\
\left(\mathrm{m}^{3} \mathrm{~kg}^{-1}\right)\end{array}$ & $\begin{array}{l}\Delta \chi \\
\text { (molar) } \\
\left(\mathrm{m}^{3} \mathrm{~mol}^{-1}\right)\end{array}$ & \\
\hline PAA (1) & $124.4^{\circ} \mathrm{C}$ & $\begin{array}{l}-4.86 \times 10^{-7} \\
\text { (vol) }\end{array}$ & $\begin{array}{l}-5.64 \times 10^{-7} \\
\text { (vol) } \\
(\text { measured } \\
-5.47 \times 10^{-7} \text { ) }\end{array}$ & $\begin{array}{l}1.17 \times 10^{-7} \\
(\mathrm{vol})\end{array}$ & $\begin{array}{l}-6.11 \times 10^{-6} \\
\text { (vol) }\end{array}$ & $\begin{array}{l}-7.09 \times 10^{-6} \\
\text { (vol) }\end{array}$ & $1.47 \times 10^{-6}$ & $1.26 \times 10^{-9}$ & $327 \times 10^{-12}$ & {$[65]^{a}$} \\
\hline PAA (2a) & $\begin{array}{l}125^{\circ} \mathrm{C} \\
\left(T_{\mathrm{NI}}=135^{\circ} \mathrm{C}\right)\end{array}$ & & & & $\begin{array}{l}-6.15 \times 10^{-9} \\
\mathrm{~m}^{3} \mathrm{~kg}^{-1} \\
\text { (mass) }\end{array}$ & $\begin{array}{l}-7.2 \times 10^{-9} \\
\mathrm{~m}^{3} \mathrm{~kg}^{-1} \\
\text { (mass) }\end{array}$ & $1.82 \times 10^{-6}$ & $1.57 \times 10^{-9}$ & $405 \times 10^{-12}$ & {$[66]^{b}$} \\
\hline PAA (2b) & $125^{\circ} \mathrm{C}$ & & & $\begin{array}{l}+1.221 \times 10^{-7} \\
\mathrm{~cm}^{3} \mathrm{~g}^{-1} \\
\text { (mass) }\end{array}$ & & & $1.78 \times 10^{-6}$ & $1.53 \times 10^{-9}$ & $395 \times 10^{-12}$ & {$[67]^{c}$} \\
\hline 7CB (1) & $\begin{array}{l}10.5^{\circ} \mathrm{C} \\
\left(T_{\mathrm{R}}=0.9\right)\end{array}$ & & & $\begin{array}{l}10.4 \times 10^{-8} \\
\text { (vol) }\end{array}$ & & & $1.31 \times 10^{-6}$ & $1.28 \times 10^{-9}$ & 356 & {$[68]^{d}$} \\
\hline & $\begin{array}{l}35.7^{\circ} \mathrm{C} \\
\left(T_{\mathrm{R}}=0.98\right)\end{array}$ & & & $\begin{array}{l}9.0 \times 10^{-8} \\
(\mathrm{vol})\end{array}$ & & 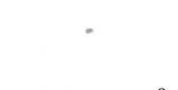 & $1.13 \times 10^{-6}$ & $1.13 \times 10^{-9}$ & 314.7 & \\
\hline 7CB (2) & $\begin{array}{l}35^{\circ} \mathrm{C} \\
\left(T_{\mathrm{R}}=0.98\right)\end{array}$ & $\begin{array}{l}-6.22 \times 10^{-7} \\
\mathrm{~cm}^{3} \mathrm{~g}^{-1}\end{array}$ & $\begin{array}{l}-6.89 \times 10^{-7} \\
\mathrm{~cm}^{3} \mathrm{~g}^{-1}\end{array}$ & $\begin{array}{l}1.0 \times 10^{-6} \\
\mathrm{~cm}^{3} \mathrm{~g}^{-1} \\
\text { (mass) }\end{array}$ & $-7.82 \times 10^{-9}$ & $-8.66 \times 10^{-9}$ & $1.25 \times 10^{-6}$ & $1.26 \times 10^{-9}$ & 349.5 & {$[69]^{e}$} \\
\hline
\end{tabular}


${ }^{a}$ Ref. [65] - These values are taken from Table II (p. 959) and were obtained by the Faraday balance method. Results are also reported from a torsion method, but these seem to be less reliable. The authors comment that $\chi_{\text {av }}$ mêsured for the nematic phase did not accord with the values measured in the solid or isotropic liquid. Hence they rejected the measured value in the nematic phase and proposed to use the value measured in the isotropic liquid, as the "trace of the susceptibility tensor should not depend on the phase in which it is measured." The conversion factor is $4 \pi$ to SI. To obtain the molar susceptibility, a value for the density of PAA is required, and this is taken from Ref. [67] as $1.160 \mathrm{~g} \mathrm{~cm}^{-3}$ at $125^{\circ} \mathrm{C}$

${ }^{b}$ Ref. [66]. The values quoted are taken from Figure 3.1 (p. 27). It is not clear if these values have been published elsewhere. The values quoted are in rationalized SI units for the mass susceptibility. The only conversion required to obtain the volume susceptibility is to multiply the mass susceptibility by the density, taken from the same author Ref. [67(see above)].

${ }^{c}$ Ref. [67] - It has been assumed that the results given in this paper (Table I, p. 278) are in cgs-emu units, and so the value given above for the SI mass susceptibility anisotropy is the cgs-emu mass susceptibility anisotropy multiplied by $4 \pi$ (to rationalize the units) $\times 10^{-3}$ to convert centimeters and grams to meters and kilograms To obtain the SI volume susceptibility anisotropy it is necessary to multiply the corresponding mass susceptibility anisotropy by the density. Values for the density of PAA over a range of temperatures are also given in the paper

${ }^{d}$ Ref. [68] - The method of measurement was using the Frederiks effect with both electric and magnetic fields, which yields a ratio of $\Delta \chi$ to $\Delta \varepsilon$. The experimental results so obtained for $\Delta \chi$ for $7 \mathrm{CB}$ do not depend on any density measurement, although a value for the density is necessary to obtain a mass or molar susceptibility. The authors assume a density for $7 \mathrm{CB}$ of $1 \mathrm{~g} \mathrm{~cm}^{-3}$, but in converting the values in the table, we have used experimental densities given in Ref. [70]. The conversion factor for cgs-emu volume susceptibility to rationalized SI units is a factor of $4 \pi$.

${ }^{e}$ Ref. [69] - The technique of measurement was the Faraday-Curie balance and this yields values for the parallel component of the susceptibility. In order to obtain the anisotropy, a measurement of the average susceptibility is also required. The authors used the value measured in the isotropic phase, and assumed that it was independent of temperature. In the paper, only fitted values for the mass susceptibility anisotropy are given. In the table above, values are derived using an average value for the mass susceptibility taken from the paper. Densities were taken from Ref. [70].

${ }^{f}$ Ref. [70] - This paper gives accurate densities for 5,6,7,8,9CB as functions of temperature.

g Ref. [71] - The method used for these measurements was the Faraday-Curie balance. The results given in the paper only refer to the derived mass susceptibility anisotropy, and no value is given for the average susceptibility that has been used. The value quoted in the table above was obtained from Figure 2 of the paper. It is assumed that cgs-emu units have been used. 


\section{References}

1. Papers of historical interest, including translations of the original articles by Reinitzer, Mauguin, Oseen, Zocher and Frederiks, can be found inSluckin, T.J., Dunmur, D.A., and Stegemeyer, H. (eds) (2004) Crystals that Flow, Taylor \& Francis, London.

2. For original papers, see (a) Repieva, A. and Frederiks, V. (1927) On the problem of the nature of the anisotropic state of matter. Zh.R.F.Kh.O, 59, 183-200 (in Russian). (b) Fréedericksz, V. and Repiewa, A. (1927) Theoretisches und experimentelles zur frage nach der natur der anisotropen flüssigkeiten. Z. Phys., 42, 532-546 (in German). (c) Fréedericksz, V. and Zolina, V. (1929) On the use of a magnetic field in the measurement of the forces tending to orient an anisotropic liquid in a thin homogeneous layer. Trans. Am. Electrochem. Soc., 55, 85-96 (in English).

3. See for example,Blinov, L.M. (2011) Structure and Properties of Liquid Crystals, Springer, New York, pp. 304-312.

4. Stewart, I.W. (2004) The Static and Dynamic Continuum Theory of Liquid Crystals, Taylor \& Francis, London.

5. Leslie, F.M. (1970) Mol. Cryst. Liq. Cryst., 12, 57-72.

6. Lee, C.H., Kwon, Y.-W., Choi, D.H., Geerts, Y.H., Koh, E., and Jin, J.-I. (2010) Adv. Mater, 22, 4405-4409.

7. Brochard, F. and de Gennes, P.-G. (1970) J. Phys., 31691.

8. Sherrell, P.L. and Crellin, D.A. (1979) J. Phys., 40, C3-211.

9. Bunning, J.D., Crellin, D.A., and Faber, T.E. (1986) Liq. Cryst., 1, 37.

10. Rohleder, J.W. and Munn, R.W. (1992) Magnetism and Optics of Molecular Crystals, John Wiley \& Sons, Ltd, Chichester, p. 27.

11. Bleaney, B. (1972) J. Magn. Reson., 8, 91.

12. Gerloch, M. and McMeeking, R.F. (1975) J.Chem. Soc., Dalton Trans., 2443.

13. Emsley, J.W. (ed.) (1983) Nuclear Magnetic Resonance of Liquid Crystals, D. Reidel, Dordrecht.

14. Dong, R. (1994) Nuclear Magnetic Resonance of Liquid Crystals, Springer-Verlag, Berlin.
15. Jiles, D. (1991) Introduction to Magnetism and Magnetic Materials, Chapman \& Hall.

16. Philo, J.S. and Fairbank, W.M. (1977) Rev. Sci. Instrum., 48, 1429.

17. Butzlaff, C., Trantwein, A.X., and Winkler, H. (1993) Meth. Enzym., 227, 412.

18. Schad, H., Baur, G., and Meier, G. (1979) J. Chem. Phys., 70, 2770.

19. de Jeu, W.H. (1980) Physical Properties of Liquid Crystalline Materials, Gordon and Breach, New York, p. 31.

20. Galyametdinov, Y.G., Haase, W., Goderis, B., Moors, D., Driesen, K., Deun, R.V., and Binnemans, K. (2007) J. Phys. Chem. B, 111, 13881-13885.

21. Galyametdinov, Y.G., Ivanova, G., Ovchinnikov, I., Prosvirin, A., Guillon, D., Heinrich, B., Dunmur, D.A., and Bruce, D.W. (1996) Liq. Cryst., 20, 831.

22. Binnemans, K., Galyametdinov, Y.G., Deun, R.V., Bruce, D.W., Collinson, S.R., Polishchuk, A.P., Bikchantaev, I., Haase, W., Prosvirin, V., Tinchurina, L., Litvinov, I., Gubajdullin, A., Rakhmatullin, A., Uytterhoeven, K., Meervelt, L.V., and Goderis, B. (2000) J. Am. Chem. Soc., 122, 4335-4344.

23. Dzhabarov, V.I., Knyazev, A.A., Nikolaev, V.F., and Galyametdinov, Y.G. (2011) Russ. J. Phys. Chem., 85, 1450-1453.

24. Bruce, D.W. (1993) J. Chem. Soc., Dalton Trans., 2983.

25. Hudson, S.A. and Maitlis, P.M. (1993) Chem. Rev., 93, 861.

26. Serrano, J.-L., Romero, P., Marcos, M., and Alonso, P.J. (1990) J. Chem. Soc., Chem. Commun., 859.

27. Bikchantaev, I., Galyametdinov, Y., Prosvirin, A., Griesar, K., Bustamente, E.A., and Haase, W. (1995) Liq. Cryst., 18, 231.

28. Galyametdinov, Y.G., Ivanova, G.I., and Ovchinikov, I.V. (1991) Bull. Acad. Sci. USSR Div. Chem. Sci., 40, 1109.

29. Piguet, C., Bünzli, J.-C.G., Donnio, B., and Guillon, D. (2006) Chem. Commun., 3755. 
30. Mironov, V.S., Galyametdinov, Y.G., Ceulemans, A., and Binnemans, K. (2000) J. Chem. Phys., 113, 10239.

31. Mironov, V.S., Galyametdinov, Y.G., Ceulemans, A., Gorlier-Walrand, C., and Binnemans, K. (2002) J. Chem. Phys., 116, 4673.

32. Corkey, R.W. (2008) Curr. Opin. Colloid Interface Sci., 13, 288.

33. Turanov, A., Ovchinikov, I., Galyametdinov, Y., and Bruce, D.W. (2001) Liq. Cryst., 28, 845.

34. Getsis, A. and Mudring, A.-V. (2010) Z. Anorg. Allg. Chem., 636, 1726-1734.

35. Galametdinov, Y.G., Knyazev, A.A., Dzhabarov, V.I., Cardinaels, T., Driesen, K., Gorller-Walrand, C., and Binnemans, K. (2008) Adv. Mater., 20, 252-257.

36. (a) Kats, E.I. and Lebedev, V.V. (1989) Sov. Phys. JETP, 69, 1155-63. (b) Kats, E.I. and Lebedev, V.V. (1991) Mol. Cryst. Liq. Cryst., 209, 329-337.

37. Kahn, O., Pie, Y., and Journaux, Y. (1991) in Inorganic Materials (eds D.W. Bruce and D. O'Hare), John Wiley \& Sons, Ltd, Chichester, p. 61.

38. Gatteschi, D. (1994) Europhys. News, 25, 50 .

39. Takahashi, S., Takai, Y., Morimoto, H., and Sonogashira, K. (1984) J. Chem. Soc., Chem. Commun., 3.

40. Rosensweig, R.E. (1985) Ferrohydrodynamics, Cambridge University Press (republished Dover Publications, New York, 1997).

41. Bashtovoy, V. and Berkovsky, B.M. (eds) (1996) Magnetic Fluids and Applications Handbook, Begell House, Wallingford.

42. Stark, H. (2001) Phys. Rep., 351, 387.

43. Burylov, S.V. and Raikher, Y.L. (1995) Mol. Cryst. Liq. Cryst., 258, 107-122.

44. Scherer, C. and Figueiredo Neto, A.M. (2005) Braz. J. Phys., 35, 718-727.

45. See for example (a) Raikher, Y.L., Burylov, S.V., and Zaklevnykh, A.N. (1987) Sov. Phys. JETP, 64, 319-324. (b) Burylov, S.V. and Raikher, Y.L. (1993) J. Magn. Magn. Mater., 122, 62-65. (c) Burylov, S.V. and Raikher, Y.L. (1994) Phys. Rev., 50, 358-367.

46. Raikher, Y.L. (1998) in Phase Transitions in Complex Fluids (eds P. Toledano and A.M. Figueiredo Neto), World Scientific, Singapore, pp. 295-315.
47. (a) Zadorozhnii, V.I., Vasil'ev, A.N., Reshetnyak, V.Y., Thomas, K.S., and Sluckin, T.J. (2006) Europhys. Lett., 73, 408-414. (b) Zadorozhnii, V.I., Sluckin, T.J., Reshetnyak, V.Y., and Thomas, K.S. (2008) SIAM J. Appl. Maths., 68, 1688-1716.

48. Rault, J., Cladis, P.E., and Burger, J.P. (1970) Phys. Lett., A32, 199-200.

49. Hayes, C.F. (1976) Mol. Cryst. Liq. Cryst., 36, 245-253.

50. Chen, S.H. and Amer, N.M. (1983) Phys. Rev. Lett., 51, 2298.

51. See for example (a) Koneracká, M., Kellnerová, V., Kopčanský, P., and Kuczynski, T. (1995) J. Magn. Magn. Mater., 140-144, 1455-1456. (b) Potočová, I. et al. (2002) J. Magn. Magn. Mater., 252, 150-152. (c) Kopčanský, P. et al. (2005) J. Magn. Magn. Mater., 289, 101-104.

52. Kopčanský, P. et al. (2010) J. Phys. Conf. Ser., 200, 072055.

53. (a) Buluy, O., Ouskova, E., Reznikov, Y., Glushchenko, A., West, J., and Reshetnyak, V. (2002) Mol. Cryst. Liq. Cryst., 375, 81-87. (b) Buluy, O. et al (2011) Soft Matter, 7, 644-649.

54. Tyszkiewicz, C., Pustelny, T., and Nowinowski-Kruszelnicki, E. (2008) Eur. Phys. J. Spec. Top., 154, 221-224.

55. Podoliak, N. et al. (2011) Soft Matter, 7, 4742-49.

56. Liebert, L. and Martinet, A. (1979) J. Phys. Lett. (Paris), 40, L363.

57. Matuo, C.Y., Tourinho, F.A., and Figueiredo-Neto, A.M. (1993) J. Magn. Magn. Mater., 122, 53.

58. Figueiredo Neto, A., Godinho, M.H., Toth-Katona, T., and Palffy-Muhoray, P. (2005) Braz. J. Phys., 35, 184-189.

59. Berejnov, V. et al. (1998) J. Colloid Interface Sci., 199, 215-217.

60. (a) Lemaire, B.J., Davidson, P., Panine, P., and Jolivet, J.P. (2004) Phys. Rev. Lett., 93, 267801. (b) Lemaire, B.J. et al. (2004) Eur. Phys. J. E, 13, 309-319.

61. Walton, D. and Shibli, S.M. (2001) J. Magn. Magn. Mater., 226-230, 1948-1950.

62. Fabre, P., Casagrande, C., Veyssie, M., Cabuil, V., and Massart, R. (1990) Phys. Rev. Lett., 64, 539. 
63. Rožič, B. et al. (2011) Ferroelectrics, 410, 37.

64. de Gennes, P.G. and Prost, J. (1993) The Physics of Liquid Crystals, 2nd edn, Oxford University Press, Oxford, p. 119.

65. Gasparoux, H. and Prost, J. (1971) J. Phys., 32, 953.

66. De Jeu, W.H. (1980) Physical Properties of Liquid Crystalline Materials, Gordon and Breach, New York, p. 27 Figure 3.1.

67. De Jeu, W.H., Classen, W.A.P., and Spruijt, A.M.J. (1976) Mol. Cryst. Liq. Cryst., 37, 269.
68. Schad, H.P., Baur, G., and Meier, G. (1979) J. Chem. Phys., 71, 3174.

69. Sherrrell, P.L. and Crellin, D.A. (1979) J. Phys., 43, C3-211.

70. Dunmur, D.A. and Miller, W.H. (1979) J. Phys., 43, C3-141.

71. Buka, A. and de Jeu, W.H. (1982) J. Phys., 43, 361. 